\title{
A PETROV-GALERKIN FINITE ELEMENT METHOD FOR FRACTIONAL CONVECTION-DIFFUSION EQUATIONS
}

\author{
BANGTI JIN, RAYTCHO LAZAROV, AND ZHI ZHOU
}

\begin{abstract}
In this work, we develop variational formulations of Petrov-Galerkin type for one-dimensional fractional boundary value problems involving either a Riemann-Liouville or Caputo derivative of order $\alpha \in(3 / 2,2)$ in the leading term and both convection and potential terms. They arise in the mathematical modeling of asymmetric super-diffusion processes in heterogeneous media. The well-posedness of the formulations and sharp regularity pickup of the variational solutions are established. A novel finite element method is developed, which employs continuous piecewise linear finite elements and "shifted" fractional powers for the trial and test space, respectively. The new approach has a number of distinct features: It allows deriving optimal error estimates in both $L^{2}(D)$ and $H^{1}(D)$ norms; and on a uniform mesh, the stiffness matrix of the leading term is diagonal and the resulting linear system is well conditioned. Further, in the Riemann-Liouville case, an enriched FEM is proposed to improve the convergence. Extensive numerical results are presented to verify the theoretical analysis and robustness of the numerical scheme.
\end{abstract}

\section{INTRODUCTION}

In this work, we consider the following one-dimensional fractional boundary value problem (FBVP)

$$
\begin{aligned}
-{ }_{0} D_{x}^{\alpha} u+b u^{\prime}+q u & =f \quad \text { in } D=(0,1), \\
u(0)=u(1) & =0,
\end{aligned}
$$

where the source term $f$ belongs to $L^{2}(D)$ or a suitable subspace, and ${ }_{0} D_{x}^{\alpha} u$ denotes either the left-sided Riemann-Liouville or Caputo fractional derivative of order $\alpha \in(3 / 2,2)$ defined in $(2.2)$ below. The choice $\alpha \in(3 / 2,2)$ is to ensure the wellposedness of problem (1.1) in the space $L^{2}(D)$, and that the solution $u$ lies in $H_{0}^{1}(D)$ (see Section 3 for details) so that the $H^{1}(D)$ error estimate makes sense. Throughout, unless otherwise stated, we assume a convection coefficient $b \in W^{1, \infty}(D)$ and a potential coefficient $q \in L^{\infty}(D)$. For $\alpha=2$, problem (1.1) recovers the canonical steady-state convection diffusion equation.

The interest in the model (1.1) is motivated by anomalous diffusion in heterogeneous media. Often it is used to describe super-diffusion processes, in which the mean squared variance grows at a rate faster than that in a Gaussian process for normal diffusion. Microscopically, the fractional derivative describes long-range interactions among particles and large particle jumps, and the choice of the onesided derivative reflects the asymmetry of the transport process $[3,6]$. The term $b u^{\prime}$ describes convection under external flow field, with a velocity $b$. The model has found successful applications in a number of areas, e.g., magnetized plasma and subsurface flow. 
1.1. Review on existing studies. The robust simulation of the model (1.1) is challenging due to the nonlocality of the fractional derivative and limited solution regularity. In the time dependent case, the finite difference method (FDM) is predominant [17, 23, 21, 2]; see also [14] for a finite element method (FEM). Often, the stability of the schemes and their error estimates were derived by assuming a sufficiently smooth solution. In this work, we focus on the steady-state model (1.1), and review below the Riemann-Liouville and Caputo derivatives separately.

In the Riemann-Liouville case, Ervin and Roop [8] (see also [9]) gave a first variational formulation of (1.1) on the space $H_{0}^{\alpha / 2}(D)$. The coercivity of the formulation was shown under suitable conditions on the coefficients $b$ and $q$. However, in the presence of the convection term, for $\alpha \leq 3 / 2$, the variational solution generally does not solve the equation in the $L^{2}(\Omega)$ sense, due to insufficient solution regularity. A Galerkin FEM was also proposed, and error estimates were provided by assuming that the solution is smooth, which remains completely open in the general case, and that the adjoint problem has full regularity pickup, which generally does not hold.

In the absence of the convection term in (1.1), it was revisited in [13], where sharp regularity pickup was established for the first time and $H^{\alpha / 2}(D)$ and $L^{2}(D)$ error estimates, directly expressed in terms of the problem data, were provided for the Galerkin FEM. However, the $L^{2}(D)$ error estimates are suboptimal. Wang and Yang [24] developed a stable Petrov-Galerkin formulation on the space $H_{0}^{\alpha-1}(D) \times$ $H_{0}^{1}(D)$, with a variable coefficient inside the fractional derivative. It was numerically realized in [25], where an $L^{2}(D)$-error estimate was provided. The problem in $[24,25]$ does not involve lower order terms, and its extension to problem (1.1) seems nontrivial. In [26], Petrov-Galerkin formulations for initial value problems for fractional ODEs and PDEs with a Riemann-Liouville derivative in time were studied. Chen et al [5] proposed a spectral method for FBVPs of general order without any lower order term, which merits exponentially convergence in the $L^{2}(D)$ norm for suitably smooth data. However, the $L^{2}(D)$ error estimate remains suboptimal [5, Remark 5.2].

One distinct feature of FBVPs with a Riemann-Liouville derivative is that the solution is usually weakly singular, irrespective of the smoothness of the source term $f$. Thus the standard FEM converges slowly. There are several ways to improve the convergence, e.g., singularity reconstruction [15] and transformation approach [12].

The Caputo case is more delicate, and was scarcely studied. For example, for $\alpha \in(1,3 / 2]$, the existence of a solution to problem (1.1) with $f \in L^{2}(D)$ is unknown. This is reminiscent of fractional diffusion with a Caputo derivative of order $\alpha \in(0,1 / 2)$ in time [10]. The only variational formulation for the Caputo case was derived in [13]. The trial space is $H_{0}^{\alpha / 2}(D)$, but the test space involves a nonlocal constraint. The stability and sharp regularity pickup were shown, and a Galerkin FEM was proposed, with optimal $H^{\alpha / 2}(D)$ but suboptimal $L^{2}(D)$ error estimates. Recently, Stynes and Gracia [22] developed a FDM for (1.1) with a Caputo derivative and a Robin boundary condition, and derived an $L^{\infty}(D)$ rate. See also [11] for a Legendre tau method, where a suboptimal $L^{2}(D)$ error estimate was given for a smooth solution. 
1.2. Our contributions and the organization of the paper. In this work, we shall develop proper variational formulations of Petrov-Galerkin type for the model (1.1), and establish their well-posedness and sharp regularity pickup. For the choice $\alpha \in(3 / 2,2)$, the variational solution satisfies $(1.1)$ in the $L^{2}(D)$ sense. Further, we develop a novel FEM with continuous piecewise linear finite elements and "shifted" fractional powers for the test and trial space, respectively.

The new FEM has a number of distinct features. First, the choice of the FEM test space allows us to derive optimal error estimates in both $L^{2}(D)$ and $H^{1}(D)$ norms that are directly expressed in terms of the problem data. In particular, this fills an outstanding gap in the theoretical analysis of FEMs for FBVPs, for which only suboptimal $L^{2}(D)$ estimates were known. Second, on a uniform mesh, the stiffness matrix of the leading term is diagonal, and the resulting linear system is well conditioned. To the best of our knowledge, it is the first FEM with such desirable property.

In the Riemann-Liouville case, we also develop an enriched FEM based on a singularity reconstruction technique $[4,15]$ to improve the convergence, by resolving the solution singularity directly. We also derive optimal error estimates in both $H^{1}(D)$ and $L^{2}(D)$ norms, thereby improving the results in [15].

The rest of the paper is organized as follows. In Section 2 we recall preliminaries on fractional calculus. The variational formulations are developed in Section 3, where the well-posedness and sharp regularity pickup are also studied. The new FEM and its implementation details are described in Section 4, and optimal convergence rates are provided. Then we present an enriched FEM for the RiemannLiouville derivative in Section 5. Finally, in Section 6, the theoretical analysis is numerically verified by extensive experiments, including nonsmooth data. Throughout the notation $c$, with or without a subscript, denotes a generic constant, which may change at different occurrences, but it is always independent of the mesh size $h$ and the solution $u$.

\section{Preliminaries on fractional CAlCulus}

We first briefly recall some preliminary facts on fractional calculus. For any $\gamma>0$ and $f \in L^{2}(D)$ we define the left-sided Riemann-Liouville fractional integral ${ }_{0} I_{x}^{\gamma} f$ of order $\gamma$ by

$$
\left({ }_{0} I_{x}^{\gamma} f\right)(x)=\frac{1}{\Gamma(\gamma)} \int_{0}^{x}(x-t)^{\gamma-1} f(t) d t
$$

where $\Gamma(\cdot)$ is the Gamma function defined by $\Gamma(x)=\int_{0}^{\infty} t^{x-1} e^{-t} d t$ for $x>0$. Then, for any $\beta>0$ with $n-1<\beta<n, n \in \mathbb{N}$, the left-sided Riemann-Liouville and Caputo derivatives of order $\beta$ of $f \in H^{n}(D)$, denoted by ${ }_{0}^{R} D_{x}^{\beta} f$ and ${ }_{0}^{C} D_{x}^{\beta} f$, are respectively defined by $[16,19]$

$$
{ }_{0}^{R} D_{x}^{\beta} u=\frac{d^{n}}{d x^{n}}\left({ }_{0} I_{x}^{n-\beta} u\right) \quad \text { and } \quad{ }_{0}^{C} D_{x}^{\beta} u={ }_{0} I_{x}^{n-\beta}\left(\frac{d^{n} u}{d x^{n}}\right) .
$$

Analogously we define the right-sided Riemann-Liouville integral ${ }_{x} I_{1}^{\gamma} f$ by

$$
\left({ }_{x} I_{1}^{\gamma} f\right)(x)=\frac{1}{\Gamma(\gamma)} \int_{x}^{1}(t-x)^{\gamma-1} f(t) d t
$$


and the right-sided derivatives of order $\beta$ by

$$
{ }_{x}^{R} D_{1}^{\beta} u=(-1)^{n} \frac{d^{n}}{d x^{n}}\left({ }_{x} I_{1}^{n-\beta} u\right) \quad \text { and } \quad{ }_{x}^{C} D_{1}^{\beta} u=(-1)^{n}{ }_{x} I_{1}^{n-\beta}\left(\frac{d^{n} u}{d x^{n}}\right) .
$$

The following formula for change of integration order is valid [16, pp. 76, Lemma $2.7]$

$$
\left({ }_{0} I_{x}^{\gamma} \psi, \varphi\right)=\left(\psi,{ }_{x} I_{1}^{\gamma} \varphi\right) \quad \forall \psi, \varphi \in L^{2}(D),
$$

where $(\cdot, \cdot)$ denotes the $L^{2}(D)$ inner product.

Now we introduce some function spaces. For any $\beta \geq 0$, we denote $H^{\beta}(D)$ to be the Sobolev space of order $\beta$ on the unit interval $D[1]$, and $\widetilde{H}^{\beta}(D)$ the set of functions in $H^{\beta}(D)$ whose extension by zero to $\mathbb{R}$ is in $H^{\beta}(\mathbb{R})$. Likewise, we define $\widetilde{H}_{L}^{\beta}(D)$ (respectively, $\widetilde{H}_{R}^{\beta}(D)$ ) to be the set of functions $u$ whose extension by zero, denoted by $\tilde{u}$, is in $H^{\beta}(-\infty, 1)$ (respectively, $H^{\beta}(0, \infty)$ ). Further, for $u \in \widetilde{H}_{L}^{\beta}(D)$, we set $\|u\|_{\widetilde{H}_{L}^{\beta}(D)}:=\|\tilde{u}\|_{H^{\beta}(-\infty, 1)}$, and similarly the norm in $\widetilde{H}_{R}^{\beta}(D)$.

The next theorem collects some useful properties of fractional integral and differential operators (see [16, pp. 73, Lemma 2.3] [13, Theorems 2.1 and 3.1]).

Theorem 2.1. The following statements hold.

(a) The integral operators ${ }_{0} I_{x}^{\beta}$ and ${ }_{x} I_{1}^{\beta}$ satisfy the semigroup property.

(b) The operators ${ }_{0}^{R} D_{x}^{\beta}$ and ${ }_{x}^{R} D_{1}^{\beta}$ extend continuously to bounded operators from $\widetilde{H}_{L}^{\beta}(D)$ and $\widetilde{H}_{R}^{\beta}(D)$, respectively, to $L^{2}(D)$.

(c) For any $s, \beta \geq 0$, the operator ${ }_{0} I_{x}^{\beta}$ is bounded from $\widetilde{H}_{L}^{s}(D)$ to $\widetilde{H}_{L}^{\beta+s}(D)$, and ${ }_{x} I_{1}^{\beta}$ is bounded from $\widetilde{H}_{R}^{s}(D)$ to $\widetilde{H}_{R}^{\beta+s}(D)$.

We shall also need the following two results. The first asserts the equivalence of the two fractional derivatives on suitable function spaces, and the second gives an algebraic property of fractional-order Sobolev spaces.

Lemma 2.1 ([13], Lemma 4.1). For $u \in \widetilde{H}_{L}^{1}(D)$ and $\beta \in(0,1),{ }_{0}^{R} D_{x}^{\beta} u={ }_{0} I_{x}^{1-\beta}\left(u^{\prime}\right)$. Similarly, for $u \in \widetilde{H}_{R}^{1}(D)$ and $\beta \in(0,1),{ }_{x}^{R} D_{1}^{\beta} u=-{ }_{x} I_{1}^{1-\beta}\left(u^{\prime}\right)$.

Lemma 2.2 ([13], Lemma 4.6). Let $0<s \leq 1, s \neq 1 / 2$. Then for any $u \in$ $\widetilde{H}^{s}(D) \cap L^{\infty}(D)$ and $v \in H^{s}(D) \cap L^{\infty}(D)$, the product $u v$ is in $\widetilde{H}^{s}(D)$.

\section{VARIATIONAL FORMULATION AND REGULARITY}

Now we develop proper variational formulations for problem (1.1), and establish their stability and sharp regularity pickup. We shall discuss the Riemann-Liouville and Caputo cases separately.

3.1. Variational formulation in the Riemann-Liouville case. First we consider the case $b, q \equiv 0$. Then it was shown in [13, Section 3] that for $f \in L^{2}(D)$, the solution $u$ of (1.1) is given by

$$
u=-{ }_{0} I_{x}^{\alpha} f+\left({ }_{0} I_{x}^{\alpha} f\right)(1) x^{\alpha-1} \in \widetilde{H}_{L}^{\alpha-1+\beta}(D),
$$

for any $\beta \in[2-\alpha, 1 / 2)$. Thus for $\alpha \in(3 / 2,2), u \in \widetilde{H}^{1}(D)$. Further, for $\varphi \in C_{0}^{\infty}(D)$, by $(2.3)$ and Lemma 2.1 , there holds

$$
\begin{aligned}
\left({ }_{0}^{R} D_{x}^{\alpha} u, \varphi\right) & =\left(\left({ }_{0} I_{x}^{2-\alpha} u\right)^{\prime \prime}, \varphi\right)=-\left(\left({ }_{0} I_{x}^{2-\alpha} u\right)^{\prime}, \varphi^{\prime}\right) \\
& =-\left({ }_{0} I_{x}^{2-\alpha} u^{\prime}, \varphi^{\prime}\right)=-\left(u^{\prime},{ }_{x} I_{1}^{2-\alpha} \varphi^{\prime}\right)=\left(u^{\prime},{ }_{x}^{R} D_{1}^{\alpha-1} \varphi\right) .
\end{aligned}
$$


This motivates us to define a bilinear form $a(\cdot, \cdot): \widetilde{H}^{1}(D) \times \widetilde{H}^{\alpha-1}(D) \rightarrow \mathbb{R}$ by

$$
a(u, \varphi):=-\left(u^{\prime},{ }_{x}^{R} D_{1}^{\alpha-1} \varphi\right) .
$$

Throughout, we set $U=\widetilde{H}^{1}(D)$ and $V=\widetilde{H}^{\alpha-1}(D)$ below, and denote by $U^{*}$ etc. the dual space of $U$ etc., and the norms on $U$ etc. by $\|\cdot\|_{U}$ etc. Further, we also denote the duality pairing by $\langle\cdot, \cdot\rangle$.

Now we state our first result on the stability of the variational formulation.

Lemma 3.1. The bilinear form $a(\cdot, \cdot)$ in (3.2) satisfies the inf-sup condition:

$$
\sup _{\varphi \in V} \frac{a(u, \varphi)}{\|\varphi\|_{V}} \geq c_{0}\|u\|_{U}
$$

Proof. For any fixed $u \in U$, let $\varphi_{u}={ }_{x}^{R} D_{1}^{2-\alpha} u-\left({ }_{x}^{R} D_{1}^{2-\alpha} u\right)(0)(1-x)^{\alpha-1}$. Clearly, $\varphi_{u}(0)=\varphi_{u}(1)=0$. For $\beta \in[2-\alpha, 1 / 2)$, the term $(1-x)^{\alpha-1} \in \widetilde{H}_{R}^{\alpha-1+\beta}(D)$. Meanwhile, by Lemma 2.1 and Theorem 2.1(c), for $u \in U$, there holds

$$
\left\|{ }_{x}^{R} D_{1}^{2-\alpha} u\right\|_{H^{\alpha-1}(D)}=\left\|I_{1}^{\alpha-1} u^{\prime}\right\|_{H^{\alpha-1}(D)} \leq c\|u\|_{U},
$$

and thus $\varphi_{u} \in V$ and is a valid test function. Further,

$$
\begin{aligned}
\left\|\varphi_{u}\right\|_{V} & \leq c\left(\left\|{ }_{x}^{R} D_{1}^{2-\alpha} u\right\|_{H^{\alpha-1}(D)}+\left.c\right|_{x} ^{R} D_{1}^{2-\alpha} u(0) \mid\left\|(1-x)^{\alpha-1}\right\|_{H^{\alpha-1}(D)}\right) \\
& \leq c\left(\|u\|_{U}+\left|\left({ }_{x} I_{1}^{\alpha-1} u^{\prime}\right)(0)\right|\right) \leq c\|u\|_{U} .
\end{aligned}
$$

Since $u(0)=u(1)=0,\left(u^{\prime},{ }_{x}^{R} D_{1}^{\alpha-1}(1-x)^{\alpha-1}\right)=c_{\alpha}\left(u^{\prime}, 1\right)=0$, and we derive the following inf-sup condition:

$$
\sup _{\varphi \in V} \frac{a(u, \varphi)}{\|\varphi\|_{V}} \geq \frac{-\left(u^{\prime},{ }_{x}^{R} D_{1}^{\alpha-1} \varphi_{u}\right)}{\left\|\varphi_{u}\right\|_{V}} \geq c_{0} \frac{-\left(u^{\prime},{ }_{x}^{R} D_{1}^{\alpha-1}\left(-{ }_{x} I_{1}^{\alpha-1} u^{\prime}\right)\right)}{\|u\|_{U}}=c_{0}\|u\|_{U} .
$$

For any nonzero $\varphi \in V$, we choose $u_{\varphi}={ }_{x} I_{1}^{2-\alpha} \varphi-\left({ }_{x} I_{1}^{2-\alpha} \varphi\right)(0)(1-x)$, which is nonzero and belongs to $U$. Then

$$
a\left(u_{\varphi}, \varphi\right)=-\left(u_{\varphi}^{\prime},{ }_{x}^{R} D_{1}^{\alpha-1} \varphi\right)=\left\|u_{\varphi}\right\|_{U}^{2}>0 .
$$

It implies that if $a(u, \varphi)=0$ for all $u \in U$, then $\varphi=0$. This and Lemma 3.1 give the stability of the variational problem for the case $b, q \equiv 0$. Namely, given any $F \in V^{*}$, there exists a unique solution $u \in U$ such that

$$
a(u, \varphi)=\langle F, \varphi\rangle \quad \forall \varphi \in V .
$$

We now turn to the general case $b, q \not \equiv 0$. The corresponding variational formulation reads: given any $F \in V^{*}$, find $u \in U$ such that

$$
A(u, \varphi)=\langle F, \varphi\rangle \quad \forall \varphi \in V,
$$

where the bilinear form $A(\cdot, \cdot): U \times V \rightarrow \mathbb{R}$ is defined by

$$
A(u, \varphi)=a(u, \varphi)+\left(b u^{\prime}, \varphi\right)+(q u, \varphi) .
$$

To study the bilinear form $A(\cdot, \cdot)$, we make the following uniqueness assumption.

Assumption 3.1. Let the bilinear form $A(\cdot, \cdot): U \times V \rightarrow \mathbb{R}$ satisfy

(a) The problem of finding $u \in U$ such that $A(u, \varphi)=0$ for all $\varphi \in V$ has only the trivial solution $u \equiv 0$.

$\left(\mathrm{a}^{*}\right)$ The problem of finding $\varphi \in V$ such that $A(u, \varphi)=0$ for all $u \in U$ has only the trivial solution $\varphi \equiv 0$. 
Theorem 3.1. Let $b, q \in L^{\infty}(D)$ and Assumption 3.1 hold. Then for any $F \in V^{*}$, there exists a unique solution $u \in U$ to problem (3.4).

Proof. In case of $b, q \equiv 0$, the assertion follows from Lemma 3.1. In general, the proof is based on Petree-Tartar's lemma [7, pp. 469, Lemma A.38]. To this end, we define two operators $S \in \mathcal{L}\left(U ; V^{*}\right)$ and $T \in \mathcal{L}\left(U ; V^{*}\right)$ by

$$
\langle S u, \varphi\rangle=A(u, \varphi) \quad \text { and } \quad(T u, \varphi)=-\left(b u^{\prime}, \varphi\right)-(q u, \varphi),
$$

respectively. By Assumption 3.1(a), the operator $S$ is injective. The compactness of the operator $T$ follows from $b, q \in L^{\infty}(D)$ and the compact embedding from $L^{2}(D)$ into $V^{*}$. Further, by Lemma 3.1, we deduce that for any $u \in U$

$$
\begin{aligned}
\|u\|_{U} & \leq c \sup _{\varphi \in V} \frac{a(u, \varphi)}{\|\varphi\|_{V}} \leq c \sup _{\varphi \in V} \frac{A(u, \varphi)}{\|\varphi\|_{V}}+c \sup _{\varphi \in V} \frac{-\left(b u^{\prime}, \varphi\right)-(q u, \varphi)}{\|\varphi\|_{V}} \\
& =c\left(\|S u\|_{V^{*}}+\|T u\|_{V^{*}}\right) .
\end{aligned}
$$

Then by Petree-Tartar's lemma, the image of the operator $S$ is closed; equivalently, there exists a constant $c_{0}>0$ such that

$$
c_{0}\|u\|_{U} \leq \sup _{\varphi \in V} \frac{A(u, \varphi)}{\|\varphi\|_{V}} \quad \forall u \in U .
$$

This and Assumption 3.1( $\left(a^{*}\right)$ show that the operator $S: U \rightarrow V^{*}$ is bijective, i.e., there exists a unique solution $u \in U$ to problem (3.4).

Theorem 3.2. Let $b, q \in L^{\infty}(D)$ and $\langle F, \varphi\rangle=(f, \varphi)$ for some $f \in L^{2}(D)$, and Assumption 3.1 hold. Then there exists a unique solution $u \in \widetilde{H}_{L}^{\alpha-1+\beta}(D) \cap \widetilde{H}^{1}(D)$ to problem (3.4) for any $\beta \in[2-\alpha, 1 / 2)$ and it satisfies

$$
\|u\|_{\widetilde{H}_{L}^{\alpha-1+\beta}(D)} \leq c\|f\|_{L^{2}(D)} .
$$

Proof. By Theorem 3.1, we have $\|u\|_{\widetilde{H}^{1}(D)} \leq c\|f\|_{L^{2}(D)}$. Then we rewrite (3.4) as $-{ }_{0}^{R} D_{x}^{\alpha} u=\tilde{f}$ with $\tilde{f}=f-b u^{\prime}-q u$. Since $b, q \in L^{\infty}(D)$ and $u \in \widetilde{H}^{1}(D)$, $b u^{\prime}+q u \in L^{2}(D)$, and $\tilde{f} \in L^{2}(D)$. Then (3.6) follows from (3.1) and Theorem $2.1(\mathrm{c})$.

Next we consider the adjoint problem: for a given $F \in U^{*}$, find $w \in V$ such that

$$
A(\varphi, w)=\langle\varphi, F\rangle \quad \forall \varphi \in U .
$$

The inf-sup condition for problem (3.7) with $b, q \equiv 0$ was shown in [24, Theorem 5.5]. The stability for $b \in W^{1, \infty}(D)$ and $q \in L^{\infty}(D)$ follows from Assumption 3.1 and the argument in the proof of Theorem 3.1. If $\langle\varphi, F\rangle=(\varphi, f)$ for some $f \in L^{2}(D)$, the solution $w$ for $b, q \equiv 0$ is given by $w=-{ }_{x} I_{1}^{\alpha} f+\left({ }_{x} I_{1}^{\alpha} f\right)(0)(1-x)^{\alpha-1}$. This implies $w \in \widetilde{H}_{R}^{\alpha-1+\beta}(D)$ with $\beta \in[2-\alpha, 1 / 2)$.

To rigorously analyze the adjoint problem, we first extend the domain of the operator ${ }_{x} I_{1}^{\gamma}$ to the space $\widetilde{H}^{-\gamma}(D), \gamma \in(0,1 / 2)$, the dual space of $\widetilde{H}^{\gamma}(D) \equiv H^{\gamma}(D)$, by means of duality (see [18] for an in depth treatment). Specifically, we define ${ }_{x} I_{1}^{\gamma}$ on $\widetilde{H}^{-\gamma}(D)$ by $\left({ }_{x} I_{1}^{\gamma} \varphi, \psi\right):=\left\langle\varphi,{ }_{0} I_{x}^{\gamma} \psi\right\rangle$ for all $\varphi \in \widetilde{H}^{-\gamma}(D), \psi \in L^{2}(D)$, and for $\alpha>\gamma,{ }_{x} I_{1}^{\alpha} \varphi:={ }_{x} I_{1}^{\alpha-\gamma}{ }_{x} I_{1}^{\gamma} \varphi$ for all $\varphi \in \widetilde{H}^{-\gamma}(D)$. Next we verify the consistency relation for $\alpha \in(3 / 2,2)$

$$
{ }_{x}^{R} D_{1 x}^{\alpha} I_{1}^{\alpha} \varphi=\varphi \in \widetilde{H}^{-\gamma}(D) \text { with } \gamma \in(0,1 / 2) .
$$


In fact, for any $\psi \in C_{0}^{\infty}(D)$, by Theorem 2.1(a) and (2.3), there holds

$$
\begin{aligned}
\left\langle{ }_{x}^{R} D_{1}^{\alpha}{ }_{x} I_{1}^{\alpha} \varphi, \psi\right\rangle & =\left\langle\left({ }_{x} I_{1}^{2-\alpha}{ }_{x} I_{1}^{\alpha} \varphi\right)^{\prime \prime}, \psi\right\rangle \stackrel{\text { def }}{=}\left\langle\left({ }_{x} I_{1}^{2-\gamma}{ }_{x} I_{1}^{\gamma} \varphi\right)^{\prime \prime}, \psi\right\rangle=\left\langle{ }_{x} I_{1}^{2-\gamma}{ }_{x} I_{1}^{\gamma} \varphi, \psi^{\prime \prime}\right\rangle \\
& =\left({ }_{x} I_{1}^{\gamma} \varphi,{ }_{0} I_{x}^{2-\gamma} \psi^{\prime \prime}\right) \stackrel{\text { def }}{=}\left\langle\varphi,{ }_{0} I_{x}^{2} \psi^{\prime \prime}\right\rangle=\langle\varphi, \psi\rangle .
\end{aligned}
$$

Now we show that, for $\alpha>\gamma>0$, the operator ${ }_{x} I_{1}^{\alpha}$ is bounded from $\widetilde{H}^{-\gamma}(D)$ to $\widetilde{H}_{R}^{\alpha-\gamma}(D)$. Indeed, by Theorem 2.1(a) and (c), we have

$$
\begin{aligned}
\left\|{ }_{x} I_{1}^{\alpha} \varphi\right\|_{\widetilde{H}_{R}^{\alpha-\gamma}(D)}: & =\left\|{ }_{x} I_{1}^{\alpha-\gamma}{ }_{x} I_{1}^{\gamma} \varphi\right\|_{\widetilde{H}_{R}^{\alpha-\gamma}(D)} \leq c\left\|_{x} I_{1}^{\gamma} \varphi\right\|_{L^{2}(D)} \\
& =c \sup _{\psi \in L^{2}(D)} \frac{\left\langle\varphi,{ }_{0} I_{x}^{\gamma} \psi\right\rangle}{\|\psi\|_{L^{2}(D)}(D)} \leq c\|\varphi\|_{\widetilde{H}^{-\gamma}(D)} .
\end{aligned}
$$

Thus the representation $w=-{ }_{x} I_{1}^{\alpha} f+\left({ }_{x} I_{1}^{\alpha} f\right)(0)(1-x)^{\alpha-1}$ is a solution to (3.7) with $b, q \equiv 0$ and $F=f \in \widetilde{H}^{-\gamma}(D), \gamma \in(0,1 / 2)$. In sum, we have the following lemma.

Lemma 3.2. Let $F=f \in \widetilde{H}^{-\gamma}(D), \gamma \in(0,1 / 2)$, and $b, q \equiv 0$. Then $w=$ $-{ }_{x} I_{1}^{\alpha} f+\left({ }_{x} I_{1}^{\alpha} f\right)(0)(1-x)^{\alpha-1}$ is a solution of problem (3.7) and for $\beta \in[2-\alpha, 1 / 2)$

$$
\|w\|_{\widetilde{H}_{R}^{\alpha-1+\beta}(D)} \leq c\|f\|_{\widetilde{H}^{-\gamma}(D)} .
$$

Now we can state a regularity result for the adjoint problem (3.7).

Theorem 3.3. Let $b \in W^{1, \infty}(D), q \in L^{\infty}(D)$ and $\langle F, \varphi\rangle=(f, \varphi)$ for some $f \in L^{2}(D)$ and Assumption 3.1 hold. Then there exists a unique solution $w \in$ $\widetilde{H}_{R}^{\alpha-1+\beta}(D) \cap \widetilde{H}^{\alpha-1}(D)$ to problem (3.7) for any $\beta \in[2-\alpha, 1 / 2)$ and it satisfies

$$
\|w\|_{\widetilde{H}_{R}^{\alpha-1+\beta}(D)} \leq c\|f\|_{L^{2}(D)} .
$$

Proof. By the inf-sup condition, there exists a solution $w \in \widetilde{H}^{\alpha-1}(D)$ to (3.7). Next we rewrite it into $a(\varphi, w)=\langle\varphi, \widetilde{f}\rangle$, with $\widetilde{f}=f+(b w)^{\prime}-q w$, with $\|\widetilde{f}\|_{\widetilde{H}^{\alpha-2}(D)} \leq$ $c\|f\|_{L^{2}(D)}$. By Lemma 3.2, $w=-{ }_{x} I_{1}^{\alpha} \tilde{f}+\left({ }_{x} I_{1}^{\alpha} \widetilde{f}\right)(0)(1-x)^{\alpha-1}$. Since $\alpha>3 / 2$, by Theorem 2.1(c), ${ }_{x} I_{1}^{\alpha} \widetilde{f} \in \widetilde{H}_{R}^{2 \alpha-2}(D) \subset \widetilde{H}_{R}^{\alpha-1+\beta}(D)$, for any $\beta \in[2-\alpha, 1 / 2)$. Hence, the desired estimate holds: $\|w\|_{\widetilde{H}_{R}^{\alpha-1+\beta}(D)} \leq c\|\widetilde{f}\|_{\widetilde{H}^{\alpha-2}(D)} \leq c\|f\|_{L^{2}(D)}$.

3.2. Variational formulation in the Caputo case. Now we consider the Caputo case. For $b, q \equiv 0$, the solution of (1.1) is given by [13, Section 3]

$$
u=-{ }_{x} I_{1}^{\alpha} f+\left({ }_{x} I_{1}^{\alpha} f\right)(0) x \in H^{\alpha}(D) \cap \widetilde{H}^{1}(D) .
$$

Recall the defining relation ${ }_{0}^{C} D_{x}^{\alpha} u={ }_{0}^{R} D_{x}^{\alpha}\left(u-u^{\prime}(0) x-u(0)\right)$ [16, pp. 91]. Hence, with $u(0)=0$, for any $\varphi \in \widetilde{H}_{R}^{\infty}(D)$, upon integration by parts, we have

$$
\left({ }_{0}^{C} D_{x}^{\alpha} u, \varphi\right)=\left({ }_{0}^{R} D_{x}^{\alpha}\left(u-u^{\prime}(0) x\right), \varphi\right)=\left(u^{\prime},{ }_{x}^{R} D_{1}^{\alpha-1} \varphi\right)-u^{\prime}(0)\left({ }_{0}^{R} D_{x}^{\alpha} x, \varphi\right) .
$$

Since $u^{\prime}(0)$ does not make sense on the space $\widetilde{H}^{1}(D)$, we choose the test function $\varphi$ such that $\left({ }_{0}^{R} D_{x}^{\alpha} x, \varphi\right)=0$, i.e., $\left(x^{1-\alpha}, \varphi\right)=0$. Hence, we let $W=\left\{\varphi \in \widetilde{H}_{R}^{\alpha-1}(D)\right.$ : $\left.\left(\varphi, x^{1-\alpha}\right)=0\right\}$, and introduce a bilinear form $a(\cdot, \cdot): U \times W \rightarrow \mathbb{R}$ by

$$
a(u, \varphi)=-\left(u^{\prime},{ }_{x}^{R} D_{1}^{\alpha-1} \varphi\right) .
$$

The only difference from the Riemann-Liouville case lies in the test space $W$ : in the Caputo case, it involves an integral constraint $\left(\varphi, x^{1-\alpha}\right)=0$.

The following lemma gives the stability of the bilinear form. 
Lemma 3.3. The bilinear form $a(\cdot, \cdot)$ in (3.10) satisfies

$$
\sup _{\varphi \in W} \frac{a(u, \varphi)}{\|\varphi\|_{W}} \geq c_{0}\|u\|_{U}
$$

Proof. For a fixed $u \in \widetilde{H}^{1}(D)$, let $\varphi_{u}=-{ }_{x} I_{1}^{\alpha-1} u^{\prime}+c_{1}(1-x)^{\alpha-1}$, where $c_{1}$ is chosen such that $\left(\varphi_{u}, x^{1-\alpha}\right)=0$, i.e., $c_{1}=-\left({ }_{x}^{R} D_{1}^{2-\alpha} u, x^{1-\alpha}\right) /\left((1-x)^{\alpha-1}, x^{1-\alpha}\right)$. Since $u^{\prime} \in L^{2}(D)$ and $(1-x)^{\alpha-1} \in \widetilde{H}_{R}^{\alpha-1+\beta}(D)$ with $\beta \in[2-\alpha, 1 / 2)$, by Theorem 2.1(c), we have $\varphi_{u} \in W$. Further, by Theorem 2.1,

$$
\left\|\varphi_{u}\right\|_{W} \leq c\left(\left\|_{x} I_{1}^{\alpha-1} u^{\prime}\right\|_{\widetilde{H}_{R}^{\alpha-1}(D)}+\left\|_{x} I_{1}^{\alpha-1} u^{\prime}\right\|_{L^{\infty}(D)}\right) \leq c\|u\|_{U} .
$$

The desired assertion follows from the argument in the proof of Lemma 3.1.

For any $\varphi \neq 0 \in W$, let $u_{\varphi}={ }_{x} I_{1}^{2-\alpha} \varphi$. Since $\varphi \in W,\left({ }_{x} I_{1}^{2-\alpha} \varphi\right)(0)=0$, and obviously $u_{\varphi}(1)=0$, we deduce $u_{\varphi} \in U$. Further,

$$
a\left(u_{\varphi}, \varphi\right)=-\left(u_{\varphi}^{\prime},{ }_{x}^{R} D_{1}^{\alpha-1} \varphi\right)=\left\|{ }_{x}^{R} D_{1}^{\alpha-1} \varphi\right\|_{L^{2}(D)}^{2}=\|\varphi\|_{W}^{2}>0 .
$$

Hence if $a(u, \varphi)=0$ for all $u \in U$, then $\varphi=0$. This and Lemma 3.3 imply the variational stability. In the case $b, q \neq 0$, the variational formulation reads: given any $F \in W^{*}$, find $u \in U$ such that

$$
A(u, \varphi)=\langle F, \varphi\rangle \quad \forall \varphi \in W,
$$

where the bilinear form $A(\cdot, \cdot): U \times W \rightarrow \mathbb{R}$ is given by

$$
A(u, \varphi)=a(u, \varphi)+\left(b u^{\prime}, \varphi\right)+(q u, \varphi) .
$$

To analyze problem (3.13), we assume the unique solvability.

Assumption 3.2. Let the bilinear form $A(\cdot, \cdot): U \times W \rightarrow \mathbb{R}$ satisfy

(a) The problem of finding $u \in U$ such that $A(u, \varphi)=0$ for all $\varphi \in W$ has only the trivial solution $u \equiv 0$.

$\left(\mathrm{a}^{*}\right)$ The problem of finding $\varphi \in W$ such that $A(u, \varphi)=0$ for all $u \in U$ has only the trivial solution $\varphi \equiv 0$.

Under Assumption 3.2, we have the following existence result. The proof is identical to that of Theorem 3.1 and hence omitted.

Theorem 3.4. Let Assumption 3.2 hold and $b, q \in L^{\infty}(D)$. Then for any $F \in W^{*}$, there exists a unique solution $u \in U$ to (3.13).

Theorem 3.5. Let Assumption 3.2 hold and $s \in[0,1 / 2)$. If $\langle F, v\rangle=(f, v)$ for some $f \in H^{s}(D)$, and $b, q \in L^{\infty}(D) \cap H^{s}(D)$, then the solution $u \in U$ of (3.13) is in $H^{\alpha+s}(D) \cap \widetilde{H}^{1}(D)$ and further it satisfies

$$
\|u\|_{H^{\alpha+s}(D)} \leq c\|f\|_{H^{s}(D)} .
$$

Proof. We show the regularity, by rewriting (3.13) as $-{ }_{0}^{C} D_{x}^{\alpha} u=\widetilde{f}$, with $\widetilde{f}=$ $f-b u^{\prime}-q u$. By Lemma 2.2, $b u^{\prime} \in L^{2}(D)$ and $q u \in H^{s}(D)$, and thus $\tilde{f} \in L^{2}(D)$. By (3.9), $u \in H^{\alpha}(D) \cap \widetilde{H}^{1}(D)$, and by Lemma 2.2, bu $u^{\prime}$ and $q u \in H^{s}(D)$, and thus $\tilde{f} \in \widetilde{H}^{s}(D)$. The assertion follows, by appealing again to Theorem 2.1 and (3.9).

Remark 3.1. In the Riemann-Liouville case, the solution is limited to $H^{\alpha-1+\beta}(D)$, $\beta \in[2-\alpha, 1 / 2)$, irrespective of the smoothness of $f$, whereas in the Caputo case, it can be made smoother, by imposing suitable smoothness on $q, b$ and $f$. 
Now we consider the adjoint problem: given any $F \in U^{*}$, find $w \in W$ such that

$$
A(\varphi, w)=\langle\varphi, F\rangle \quad \forall \varphi \in U .
$$

The next result gives the well-posedness of the adjoint problem.

Theorem 3.6. Let Assumption 3.2 hold, $b \in W^{1, \infty}(D)$ and $q \in L^{\infty}(D)$. Then for any $F \in U^{*}$, there exists a unique solution $w \in W$ to (3.14).

Proof. First consider the case $b, q \equiv 0$. For any $w \in W,{ }_{x} I_{1}^{2-\alpha} w \in U$, and thus Theorem 2.1(a) yields

$$
w=-\left({ }_{x} I_{1}^{1} w\right)^{\prime}=-\left({ }_{x} I_{1}^{\alpha-1}{ }_{x} I_{1}^{2-\alpha} w\right)^{\prime}=-{ }_{x} I_{1}^{\alpha-1}\left({ }_{x} I_{1}^{2-\alpha} w\right)^{\prime}={ }_{x} I_{1}^{\alpha-1 R}{ }_{x} D_{1}^{\alpha-1} w .
$$

Hence by Theorem 2.1(c), for $w \in W$, there holds

$$
\|w\|_{W}=\left\|{ }_{x} I_{1}^{\alpha-1 R}{ }_{x}^{\alpha-1} w\right\|_{W} \leq c\left\|_{x}^{R} D_{1}^{\alpha-1} w\right\|_{L^{2}(D)} .
$$

Next, with $u_{w}={ }_{x} I_{1}^{2-\alpha} w \in U$ and by Theorem 2.1(c), $\left\|u_{w}\right\|_{U}=\left\|{ }_{x}^{R} D_{1}^{\alpha-1} w\right\|_{L^{2}(D)} \leq$ $c\|w\|_{W}$. Then the inf-sup condition in case of $b, q \equiv 0$ follows from (3.12) and (3.15)

$$
\sup _{u \in U} \frac{a(u, w)}{\|u\|_{U}} \geq \frac{a\left(u_{w}, w\right)}{\left\|u_{w}\right\|_{U}} \geq c_{0}\|w\|_{W}
$$

Moreover, if $u \in U$ and $a(u, w)=0$ for all $w \in W$, then $u=0$ by (3.11). This and (3.16) indicate that problem (3.14) has a unique solution $w \in W$. The wellposedness for $b, q \neq 0$ follows by repeating the argument for Theorem 3.1.

Next we derive the regularity pickup for problem (3.14). If $\langle\varphi, F\rangle=(\varphi, f)$ for some $f \in L^{2}(D)$, the strong form of the problem reads

$$
-{ }_{x}^{R} D_{1}^{\alpha} w-(b w)^{\prime}+q w=f
$$

with $w(1)=0$ and $\left(w, x^{1-\alpha}\right)=0$. For $b, q \equiv 0$, the solution $w$ is given by

$$
w=-{ }_{x} I_{1}^{\alpha} f+c_{f}(1-x)^{\alpha-1} \quad \text { with } c_{f}=\left({ }_{x} I_{1}^{\alpha} f, x^{1-\alpha}\right) /\left((1-x)^{\alpha-1}, x^{1-\alpha}\right) .
$$

The constant $c_{f}$ can be bounded by $\left|c_{f}\right| \leq c\left|\left({ }_{x} I_{1}^{\alpha} f, x^{1-\alpha}\right)\right| \leq c\|f\|_{L^{2}(D)}$. Hence $w \in$ $\widetilde{H}_{R}^{\alpha-1+\beta}(D)$ with $\beta \in[2-\alpha, 1 / 2)$. The general case can be analyzed analogously to Theorem 3.3, and then we have the following regularity result.

Theorem 3.7. Let Assumption 3.2 hold, and $b \in W^{1, \infty}(D), q \in L^{\infty}(D)$. Then with $\langle\varphi, F\rangle=(\varphi, f)$ for some $f \in L^{2}(D)$, the solution $w$ to problem (3.14) is in $\widetilde{H}^{\alpha-1}(D) \cap \widetilde{H}_{R}^{\alpha-1+\beta}(D)$ for any $\beta \in[2-\alpha, 1 / 2)$ and further it satisfies

$$
\|w\|_{\widetilde{H}_{R}^{\alpha-1+\beta}(D)} \leq c\|f\|_{L^{2}(D)} .
$$

Remark 3.2. The adjoint problem for both derivatives is of Riemann-Liouville type, but with different constraints: the Riemann-Liouville case involves $w(0)=0$, whereas the Caputo case $\left(w, x^{1-\alpha}\right)=0$. Hence, their regularity pickup is identical.

\section{Finite element approximation}

Now we apply the variational formulations to the numerical approximation of problem (1.1). We shall develop novel FEMs using continuous piecewise linear finite elements and "shifted" fractional powers $\left(x_{i}-x\right)_{+}^{\alpha-1}$ for the trial and test space, respectively, analyze their stability, and derive optimal error estimates for the approximation in the $L^{2}(D)$ and $H^{1}(D)$ norms. 
4.1. Finite element spaces and their approximation properties. First we introduce the finite element spaces based on a uniform partition of the domain $D$, with nodes $0=x_{0}<x_{1}<\ldots<x_{m}=1$ and a mesh size $h=1 / m$. We then define $U_{h}$ to be the set of functions in $U$ which are linear when restricted to the subintervals $\left[x_{i}, x_{i+1}\right], i=0, \ldots, m-1$, i.e.,

$$
U_{h}=\left\{\psi_{h} \in U: \psi_{h}=a x+b, x \in\left[x_{i}, x_{i+1}\right]\right\} .
$$

To define the test spaces, we first introduce "shifted" fractional powers

$$
\varphi_{i}(x)=\left\{\begin{array}{cc}
\left(x_{i}-x\right)^{\alpha-1} & x \leq x_{i} \\
0 & x>x_{i}
\end{array}\right\}:=\left(x_{i}-x\right)^{\alpha-1} \chi_{\left[0, x_{i}\right]}(x), \quad i=1,2, \ldots, m,
$$

where $\chi_{S}$ denotes the characteristic function of a set $S$. The basis functions $\varphi_{i}(x)$ can be written as $\varphi_{i}(x)=\Gamma(\alpha)_{x} I_{1}^{\alpha-1} \chi_{\left[0, x_{i}\right]}(x)$, i.e., the fractional derivative ${ }_{x}^{R} D_{1}^{\alpha-1} \varphi_{i}$ is piecewise constant. Clearly, $\varphi_{i} \in \widetilde{H}_{R}^{\alpha-1+\beta}(D)$ for any $\beta \in[2-\alpha, 1 / 2)$. Then we define the following finite-dimensional subspaces $V_{h} \subset V$ and $W_{h} \subset W$

$$
V_{h}=\operatorname{span}\left\{\varphi_{i}\right\}_{i=1}^{m} \cap V \quad \text { and } \quad W_{h}=\operatorname{span}\left\{\varphi_{i}\right\}_{i=1}^{m} \cap W,
$$

as the test space for the Riemann-Liouville and Caputo derivative, respectively.

Next we introduce two operators $P_{V}: L^{2}(D) \rightarrow L^{2}(D)$ and $P_{W}: L^{2}(D) \rightarrow$ $L^{2}(D)$, respectively, by: for any $\psi \in L^{2}(D)$,

$$
P_{V} \psi=\psi-\Gamma(\alpha)\left({ }_{x} I_{1}^{\alpha-1} \psi\right)(0) \quad \text { and } \quad P_{W} \psi=\psi-\left({ }_{x} I_{1}^{\alpha-1} \psi, x^{1-\alpha}\right) / \Gamma(2-\alpha) .
$$

Lemma 4.1. The operators $P_{V}$ and $P_{W}$ are bounded. Further, for $\psi \in \widetilde{H}^{1}(D)$, $P_{V}^{R} D_{1}^{\alpha-1} \psi={ }_{x}^{R} D_{1}^{\alpha-1} \psi$, and for $\psi \in W \cap \widetilde{H}_{R}^{1}(D), P_{W}{ }_{x}^{R} D_{1}^{\alpha-1} \psi={ }_{x}^{R} D_{1}^{\alpha-1} \psi$.

Proof. Let $\delta=\alpha-1$. For any $\psi \in L^{2}(D)$, the boundedness of $P_{V}$ follows from

$$
\left\|P_{V} \psi\right\|_{L^{2}(D)} \leq\|\psi\|_{L^{2}(D)}+c\left|\left({ }_{x} I_{1}^{\delta} \psi\right)(0)\right| \leq c\|\psi\|_{L^{2}(D)}
$$

The boundedness of $P_{W}$ is similar. For $\psi \in \widetilde{H}^{1}(D)$, with $\varphi_{\psi}={ }_{x}^{R} D_{1}^{\delta} \psi$, by Lemma 2.1 and Theorem 2.1(a), ${ }_{x} I_{1}^{\delta} \varphi_{\psi}=-{ }_{x} I_{1}^{\delta}\left({ }_{x} I_{1}^{1-\delta} \psi\right)^{\prime}=-\left({ }_{x} I_{1}^{1} \psi\right)^{\prime}=\psi$, and thus $\left({ }_{x} I_{1}^{\delta} \varphi_{\psi}\right)(0)=0$, which yields $P_{V} \varphi_{\psi}=\varphi_{\psi}-\Gamma(\delta+1)\left({ }_{x} I_{1}^{\delta} \varphi_{\psi}\right)(0)=\varphi_{\psi}$. Likewise, for $\psi \in W \cap \widetilde{H}_{R}^{1}(D),\left({ }_{x} I_{1}^{\delta} \varphi_{\psi}, x^{-\delta}\right)=\left(\psi, x^{-\delta}\right)=0$, which gives directly $P_{W} \varphi_{\psi}=\varphi_{\psi}$.

Now we can state important approximation properties of these spaces.

Lemma 4.2. Let the mesh be quasi-uniform and $1 \leq \gamma \leq 2$, and $\delta=\alpha-1 \in$ $(1 / 2,1)$. If $u \in H^{\gamma}(D) \cap \widetilde{H}^{1}(D)$, then

$$
\inf _{\psi_{h} \in U_{h}}\left\|u-\psi_{h}\right\|_{U} \leq c h^{\gamma-1}\|u\|_{H^{\gamma}(D)}
$$

Further, if $u \in \widetilde{H}_{R}^{\gamma}(D) \cap V$, then

$$
\inf _{\psi_{h} \in V_{h}}\left\|u-\psi_{h}\right\|_{V} \leq c h^{\min (1, \gamma-\delta)}\|u\|_{H^{\gamma}(D)} .
$$

Similarly, if $u \in \widetilde{H}_{R}^{\gamma}(D) \cap W$, then

$$
\inf _{\psi_{h} \in W_{h}}\left\|u-\psi_{h}\right\|_{W} \leq c h^{\min (1, \gamma-\delta)}\|u\|_{H^{\gamma}(D)} .
$$


Proof. Let $\Pi_{h} u \in U_{h}$ be the standard Lagrange interpolant of $u \in \widetilde{H}^{1}(D)$ so that

$$
\inf _{\psi_{h} \in U_{h}}\left\|u-\psi_{h}\right\|_{U} \leq\left\|u-\Pi_{h} u\right\|_{U}
$$

The first estimate follows from the approximation property of $\Pi_{h}[7$, Corollary 1.109 , pp. 61]. Next we consider the space $V_{h}$. For $u \in \widetilde{H}_{R}^{\gamma}(D)$, by Theorem 2.1(c), we have

$$
\left\|\left({ }_{x} I_{1}^{1-\delta} u\right)^{\prime}\right\|_{\widetilde{H}_{R}^{\gamma-\delta}(D)} \leq c\left\|_{x} I_{1}^{1-\delta} u\right\|_{\widetilde{H}_{R}^{\gamma+1-\delta}(D)} \leq c\|u\|_{\widetilde{H}_{R}^{\gamma}(D)} .
$$

Hence $\varphi_{u}:=-\left({ }_{x} I_{1}^{1-\delta} u\right)^{\prime}={ }_{x}^{R} D_{1}^{\delta} u$ belongs to $\widetilde{H}_{R}^{\gamma-\delta}(D)$. On the space $L^{2}(D)$, we define a projection operator $\Pi_{0}: L^{2}(D) \rightarrow L^{2}(D)$ by (with $h_{i}=x_{i+1}-x_{i}$ )

$$
\Pi_{0} \psi(x)=\frac{1}{h_{i}} \int_{x_{i}}^{x_{i+1}} \psi(s) d s \quad x \in\left(x_{i}, x_{i+1}\right] .
$$

By the definitions of $\Pi_{0}$ and $P_{V},{ }_{x} I_{1}^{\delta}\left(P_{V} \Pi_{0} \psi\right) \in V_{h}$ for any $\psi \in L^{2}(D)$. Then using (4.1), the property of $\Pi_{0}$ and Lemma 4.1 , we deduce

$$
\begin{aligned}
\inf _{\psi_{h} \in V_{h}}\left\|u-\psi_{h}\right\|_{V} & \leq\left\|_{x}^{R} D_{1}^{\delta}\left(u-{ }_{x} I_{1}^{\delta}\left(P_{V} \Pi_{0} \varphi_{u}\right)\right)\right\|_{L^{2}(D)}=\left\|\varphi_{u}-P_{V} \Pi_{0} \varphi_{u}\right\|_{L^{2}(D)} \\
& =\left\|P_{V}\left(\varphi_{u}-\Pi_{0} \varphi_{u}\right)\right\|_{L^{2}(D)} \leq c\left\|\varphi_{u}-\Pi_{0} \varphi_{u}\right\|_{L^{2}(D)} \\
& \leq c h^{\min (1, \gamma-\delta)}\left\|\varphi_{u}\right\|_{H^{\gamma-\delta}(D)} \leq c h^{\min (1, \gamma-\delta)}\|u\|_{\widetilde{H}_{R}^{\gamma}(D)} .
\end{aligned}
$$

Last, by the definitions of $\Pi_{0}$ and $P_{W},{ }_{x} I_{1}^{\delta}\left(P_{W} \Pi_{0} \psi\right) \in W_{h}$ for any $\psi \in L^{2}(D)$. Now the $L^{2}(D)$ stability of $P_{W}$ and the identity $P_{W} \varphi_{u}=\varphi_{u}$ from Lemma 4.1 yield

$$
\begin{aligned}
\inf _{\psi_{h} \in W_{h}}\left\|u-\psi_{h}\right\|_{W} & \leq\left\|_{x}^{R} D_{1}^{\delta}\left(u-{ }_{x} I_{1}^{\delta}\left(P_{W} \Pi_{0} \varphi_{u}\right)\right)\right\|_{L^{2}(D)}=\left\|P_{W}\left(\varphi_{u}-\Pi_{0} \varphi_{u}\right)\right\|_{L^{2}(D)} \\
& \leq c\left\|\varphi_{u}-\Pi_{0} \varphi_{u}\right\|_{L^{2}(D)} \leq c h^{\min (1, \gamma-\delta)}\|u\|_{\widetilde{H}_{R}^{\gamma}(D)} .
\end{aligned}
$$

4.2. Error estimates in the Riemann-Liouville case. The finite element problem is: given any $F \in V^{*}$, find $u_{h} \in U_{h}$ such that

$$
A\left(u_{h}, \varphi_{h}\right)=\left\langle F, \varphi_{h}\right\rangle \quad \forall \varphi_{h} \in V_{h} .
$$

We shall establish optimal error estimates for the approximation $u_{h}$ for $\langle F, v\rangle \equiv$ $(f, v)$ with $f \in L^{2}(D)$, using several technical lemmas.

A first lemma shows the stability of problem (4.3) when $b, q \equiv 0$.

Lemma 4.3. For the bilinear form $a(\cdot, \cdot)$ in $(3.2)$, there holds

$$
\sup _{\varphi_{h} \in V_{h}} \frac{a\left(\psi_{h}, \varphi_{h}\right)}{\left\|\varphi_{h}\right\|_{V}} \geq c\left\|\psi_{h}\right\|_{U} \quad \forall \psi_{h} \in U_{h}
$$

and the finite element problem: Find $u_{h} \in U_{h}$ such that

$$
a\left(u_{h}, \varphi_{h}\right)=\left(f, \varphi_{h}\right) \quad \forall \varphi_{h} \in V_{h},
$$

has a unique solution.

Proof. For any $\psi_{h} \in U_{h}$, let $\varphi_{h}=-{ }_{x} I_{1}^{\alpha-1}\left(P_{V} \psi_{h}^{\prime}\right)$. By Theorem 2.1, $\varphi_{h} \in V_{h}$ and

$$
\begin{aligned}
a\left(\psi_{h}, \varphi_{h}\right) & =-\left(\psi_{h}^{\prime},{ }_{x}^{R} D_{1}^{\alpha-1}\left(-{ }_{x} I_{1}^{\alpha-1}\left(P_{V} \psi_{h}^{\prime}\right)\right)\right)=\left(\psi_{h}^{\prime}, P_{V} \psi_{h}^{\prime}\right) \\
& =\left(\psi_{h}^{\prime}, \psi_{h}^{\prime}\right)-\Gamma(\alpha)\left({ }_{x} I_{1}^{\alpha-1} \psi_{h}^{\prime}\right)(0)\left(\psi_{h}^{\prime}, 1\right)=\left\|\psi_{h}\right\|_{U}^{2} .
\end{aligned}
$$


Further, by Lemma 4.1 and $\varphi_{h}(0)=0$, there holds

$$
\left\|\varphi_{h}\right\|_{V}=\left\|_{x} I_{1}^{\alpha-1}\left(P_{V} \psi_{h}^{\prime}\right)\right\|_{V} \leq c\left\|P_{V} \psi_{h}^{\prime}\right\|_{L^{2}(D)} \leq c\left\|\psi_{h}\right\|_{U} .
$$

Thus the condition (4.4) holds. Since the stiffness matrix is square, the existence follows from uniqueness, which is a direct consequence of (4.4), and thus problem (4.5) has a unique solution $u_{h} \in U_{h}$.

Next, we introduce the (adjoint) Ritz projection $R_{h}: V \rightarrow V_{h}$ defined by

$$
a\left(\psi_{h}, R_{h} \varphi\right)=a\left(\psi_{h}, \varphi\right) \quad \forall \psi_{h} \in U_{h} .
$$

Lemma 4.4. The operator $R_{h}$ is well-defined and satisfies for any $\beta \in(2-\alpha, 1 / 2)$

$$
\begin{aligned}
\left\|R_{h} \varphi\right\|_{V} & \leq c\|\varphi\|_{V}, \\
\left\|\varphi-R_{h} \varphi\right\|_{L^{2}(D)} & \leq c h^{\alpha-2+\beta}\|\varphi\|_{V} .
\end{aligned}
$$

Proof. For any $\varphi_{h} \in V_{h}$, let $\psi_{h}={ }_{x} I_{1}^{2-\alpha} \varphi_{h}-\left({ }_{x} I_{1}^{2-\alpha} \varphi_{h}\right)(0)(1-x)$. Then $\psi_{h}(0)=$ $\psi_{h}(1)=0$, and $\psi_{h}^{\prime}=-{ }_{x}^{R} D_{1}^{\alpha-1} \varphi_{h}+\left({ }_{x} I_{1}^{2-\alpha} \varphi_{h}\right)(0)$, i.e., $\psi_{h}$ is the primitive of a piecewise constant function, hence $\psi_{h} \in U_{h}$. Since ${ }_{x}^{R} D_{1}^{2-\alpha} \psi_{h}=\varphi_{h}-\left({ }_{x} I_{1}^{2-\alpha} \varphi_{h}\right)(0)\left({ }_{x}^{R} D_{1}^{2-\alpha}(1-\right.$ $x)$ ) and $\varphi_{h}(0)=0$, we deduce ${ }_{x}^{R} D_{1}^{2-\alpha} \psi_{h}(0)=c_{0}\left({ }_{x} I_{1}^{2-\alpha} \varphi_{h}\right)(0)$, with $c_{0}=-\left({ }_{x}^{R} D_{1}^{2-\alpha}(1-\right.$ $x)(0) \neq 0$. Thus the following bound holds

$$
\left|\left({ }_{x} I_{1}^{2-\alpha} \varphi_{h}\right)(0)\right| \leq\left. c\right|_{x} ^{R} D_{1}^{2-\alpha} \psi_{h}(0)|=c|\left({ }_{x} I_{1}^{\alpha-1} \psi_{h}^{\prime}\right)(0) \mid \leq c\left\|\psi_{h}\right\|_{U},
$$

which directly yields

$$
\left\|\varphi_{h}\right\|_{V}=\left\|{ }_{x}^{R} D_{1}^{\alpha-1} \varphi_{h}\right\|_{L^{2}(D)} \leq c\left(\left\|\psi_{h}^{\prime}\right\|_{L^{2}(D)}+c\left|\left({ }_{x} I_{1}^{2-\alpha} \varphi_{h}\right)(0)\right|\right) \leq c\left\|\psi_{h}\right\|_{U} .
$$

This and the identity $a\left(\psi_{h}, \varphi_{h}\right)=\left\|\psi_{h}\right\|_{U}^{2}$ give the following discrete inf-sup condition

$$
\sup _{\psi_{h} \in U_{h}} \frac{a\left(\psi_{h}, \varphi_{h}\right)}{\left\|\psi_{h}\right\|_{U}} \geq c\left\|\varphi_{h}\right\|_{V} \quad \forall \varphi_{h} \in V_{h}
$$

which shows that $R_{h}$ is well-defined. Then the $\widetilde{H}^{\alpha-1}(D)$-stability of $R_{h}$ follows:

$$
\left\|R_{h} \varphi\right\|_{V} \leq c \sup _{\psi_{h} \in U_{h}} \frac{a\left(\psi_{h}, R_{h} \varphi\right)}{\left\|\psi_{h}\right\|_{U}}=c \sup _{\psi_{h} \in U_{h}} \frac{a\left(\psi_{h}, \varphi\right)}{\left\|\psi_{h}\right\|_{U}} \leq c\|\varphi\|_{V} .
$$

Next let $g$ be the solution to (3.4) with $F=\varphi-R_{h} \varphi$. By Theorem 3.2, $\|g\|_{\widetilde{H}_{L}^{\alpha-1+\beta}(D)} \leq$ $c\left\|\varphi-R_{h} \varphi\right\|_{L^{2}(D)}, \beta \in(2-\alpha, 1 / 2)$. Then Galerkin orthogonality, and Lemma 4.2 give

$$
\begin{aligned}
\left\|\varphi-R_{h} \varphi\right\|_{L^{2}(D)}^{2} & =a\left(g, \varphi-R_{h} \varphi\right) \leq c \inf _{\psi_{h} \in U_{h}}\left\|g-\psi_{h}\right\|_{U}\left\|\varphi-R_{h} \varphi\right\|_{V} \\
& \leq c h^{\alpha-2+\beta}\left\|\varphi-R_{h} \varphi\right\|_{L^{2}(D)}\|\varphi\|_{V} .
\end{aligned}
$$

The next result gives the stability for the discrete variational formulation in the general case, using a kickback technique [20, 13].

Lemma 4.5. Let Assumption 3.1 hold, $f \in L^{2}(D)$, and $b, q \in L^{\infty}(D)$. Then there exists an $h_{0}>0$ such that for all $h \leq h_{0}$

$$
c\left\|\psi_{h}\right\|_{U} \leq \sup _{\varphi_{h} \in V_{h}} \frac{A\left(\psi_{h}, \varphi_{h}\right)}{\left\|\varphi_{h}\right\|_{V}} \quad \forall \psi_{h} \in U_{h} .
$$

For such $h$, the finite element problem: Find $u_{h} \in U_{h}$ such that

$$
A\left(u_{h}, \varphi_{h}\right)=\left(f, \varphi_{h}\right) \quad \forall \varphi_{h} \in V_{h},
$$


has a unique solution.

Proof. For any $\psi_{h} \in U_{h}$, by the inf-sup condition (3.5), there holds

$$
c_{0}\left\|\psi_{h}\right\|_{U} \leq \sup _{\varphi \in V} \frac{A\left(\psi_{h}, \varphi\right)}{\|\varphi\|_{V}} \leq \sup _{\varphi \in V} \frac{A\left(\psi_{h}, \varphi-R_{h} \varphi\right)}{\|\varphi\|_{V}}+\sup _{\varphi \in V} \frac{A\left(\psi_{h}, R_{h} \varphi\right)}{\|\varphi\|_{V}}=: \mathrm{I}+\mathrm{II} .
$$

By Lemma 4.4, we have for $\beta \in(1-\alpha / 2,1 / 2)$

$$
\mathrm{I}=\sup _{\varphi \in V} \frac{\left(b \psi_{h}^{\prime}+q \psi_{h}, \varphi-R_{h} \varphi\right)}{\|\varphi\|_{V}} \leq c \sup _{\varphi \in V} \frac{\left\|\psi_{h}\right\|_{U}\left\|\varphi-R_{h} \varphi\right\|_{L^{2}(D)}}{\|\varphi\|_{V}} \leq c_{1} h^{\alpha-2+\beta}\left\|\psi_{h}\right\|_{U}
$$

Meanwhile, the second term II can be bounded by (3.5) and Lemma 4.4:

$$
\mathrm{II} \leq c \sup _{\varphi \in V} \frac{A\left(\psi_{h}, R_{h} \varphi\right)}{\left\|R_{h} \varphi\right\|_{V}}=c \sup _{\varphi_{h} \in V_{h}} \frac{A\left(\psi_{h}, \varphi_{h}\right)}{\left\|\varphi_{h}\right\|_{V}} .
$$

By choosing an $h_{0}$ such that $c_{1} h_{0}^{\alpha-2+\beta}=c_{0} / 2$ we get the discrete inf-sup condition (4.7), and the unique existence of the solution to (4.8) follows directly.

Last we give some error estimates for the adjoint problem.

Lemma 4.6. Let Assumption 3.1 hold, $f \in L^{2}(D), b \in W^{1, \infty}(D)$ and $q \in L^{\infty}(D)$, and $w$ be the solution of the adjoint problem (3.7). Then there holds

$$
\inf _{\psi_{h} \in V_{h}}\left\|w-\psi_{h}\right\|_{L^{2}(D)}+\inf _{\psi_{h} \in V_{h}}\left\|w-\psi_{h}\right\|_{V} \leq \operatorname{ch}\|f\|_{L^{2}(D)} .
$$

Proof. By the solution representation, $w=w^{r}+w^{s}$ and $w^{s}=\mu(1-x)^{\alpha-1}$, where the regular part $w^{r} \in \widetilde{H}_{R}^{\alpha}(D)$, and $\mu \in \mathbb{R}$. By Theorem 3.3, there holds

$$
\begin{aligned}
\left\|w^{r}\right\|_{\widetilde{H}_{R}^{\alpha}(D)} & \leq\left\|{ }_{x} I_{1}^{\alpha} f\right\|_{\widetilde{H}_{R}^{\alpha}(D)}+\left\|{ }_{x} I_{1}^{\alpha}(b w)^{\prime}\right\|_{\widetilde{H}_{R}^{\alpha}(D)}+\left\|{ }_{x} I_{1}^{\alpha}(q w)\right\|_{\widetilde{H}_{R}^{\alpha}(D)} \\
& \leq c\left(\|f\|_{L^{2}(D)}+\|b w\|_{\widetilde{H}_{R}^{1}(D)}+\|q w\|_{L^{2}(D)}\right) \leq c\|f\|_{L^{2}(D)} .
\end{aligned}
$$

Let $\varphi_{w^{r}}={ }_{x}^{R} D_{1}^{\alpha-1} w^{r}, \varphi_{w^{s}}={ }_{x}^{R} D_{1}^{\alpha-1} w^{s}$ and $\varphi_{w}=\varphi_{w^{r}}+\varphi_{w^{s}}$. By Lemma 4.1, we have

$$
\begin{aligned}
\left\|\varphi_{w}-P_{V} \Pi_{0} \varphi_{w}\right\|_{L^{2}(D)} & =\left\|P_{V}\left(\varphi_{w}-\Pi_{0} \varphi_{w}\right)\right\|_{L^{2}(D)} \leq c\left\|\varphi_{w}-\Pi_{0} \varphi_{w}\right\|_{L^{2}(D)} \\
& \leq c\left\|\varphi_{w^{r}}-\Pi_{0} \varphi_{w^{r}}\right\|_{L^{2}(D)}+c\left\|\varphi_{w^{s}}-\Pi_{0} \varphi_{w^{s}}\right\|_{L^{2}(D)} .
\end{aligned}
$$

In view of $\varphi_{w^{r}} \in \widetilde{H}_{R}^{1}(D)$, by Lemma 4.2 and (4.10), we deduce

$$
\left\|\varphi_{w^{r}}-\Pi_{0} \varphi_{w^{r}}\right\|_{L^{2}(D)} \leq c h\left\|\varphi_{w^{r}}\right\|_{\widetilde{H}_{R}^{1}(D)} \leq c h\left\|w^{r}\right\|_{\widetilde{H}_{R}^{\alpha}(D)} \leq c h\|f\|_{L^{2}(D)} .
$$

Meanwhile since $\varphi_{w^{s}}$ is a constant, $\left\|\varphi_{w^{s}}-\Pi_{0} \varphi_{w^{s}}\right\|_{L^{2}(D)}=0$. Then by letting $\psi_{h}:={ }_{x} I_{1}^{\alpha-1} P_{V} \Pi_{0} \varphi_{w} \in V_{h}$ and Lemma 4.1, there holds

$\inf _{\psi_{h} \in V_{h}}\left\|w-\psi_{h}\right\|_{V} \leq\left\|_{x}^{R} D_{1}^{\alpha-1}\left(w-\psi_{h}\right)\right\|_{L^{2}(D)} \leq\left\|\varphi_{w}-P_{V} \Pi_{0} \varphi_{w}\right\|_{L^{2}(D)} \leq \operatorname{ch}\|f\|_{L^{2}(D)}$.

Since $w \in \widetilde{H}_{R}^{1}(D)$, there holds $w={ }_{x} I_{1}^{\alpha-1}{ }_{x} D_{1}^{\alpha-1} w={ }_{x} I_{1}^{\alpha-1} \varphi_{w}$. Hence

$$
\begin{aligned}
\inf _{\psi_{h} \in V_{h}}\left\|w-\psi_{h}\right\|_{L^{2}(D)} & \leq\left\|I_{1}^{\alpha-1}\left(\varphi_{w}-P_{V} \Pi_{0} \varphi_{w}\right)\right\|_{L^{2}(D)} \\
& \leq c\left\|\varphi_{w}-P_{V} \Pi_{0} \varphi_{w}\right\|_{L^{2}(D)} \leq c h\|f\|_{L^{2}(D)} .
\end{aligned}
$$

Remark 4.1. The $L^{2}(D)$ estimate in Lemma 4.6 is suboptimal, but the $H^{\alpha-1}(D)$ estimate suffices deriving an optimal $L^{2}(D)$ estimate in Theorem 4.1. 
Now, we state the main theorem of this part, i.e., optimal error estimates in the $L^{2}(D)$ and $H^{1}(D)$ norms for the Petrov-Galerkin FEM in the Riemann-Liouville case.

Theorem 4.1. Let Assumption 3.1 hold, $f \in L^{2}(D), b \in W^{1, \infty}(D)$, and $q \in$ $L^{\infty}(D)$. Then there exists an $h_{0}>0$ such that for all $h \leq h_{0}$, the solution $u_{h} \in U_{h}$ to problem (4.8) satisfies for any $\beta \in(2-\alpha, 1 / 2)$,

$$
\left\|u-u_{h}\right\|_{L^{2}(D)}+h\left\|u-u_{h}\right\|_{U} \leq c h^{\alpha-1+\beta}\|f\|_{L^{2}(D)} .
$$

Proof. The error estimate in the $\widetilde{H}^{1}(D)$-norm follows from Cea's lemma, (4.7) and Galerkin orthogonality. Specifically, for any $h \leq h_{0}$ and $\psi_{h} \in U_{h}$, by (4.7)

$$
\left\|u_{h}-\psi_{h}\right\|_{U} \leq c \sup _{\varphi_{h} \in V_{h}} \frac{A\left(u_{h}-\psi_{h}, \varphi_{h}\right)}{\left\|\varphi_{h}\right\|_{V}} \leq c \sup _{\varphi_{h} \in V_{h}} \frac{A\left(u-\psi_{h}, \varphi_{h}\right)}{\left\|\varphi_{h}\right\|_{V}} \leq c\left\|u-\psi_{h}\right\|_{U} .
$$

Hence the triangle inequality yields for any $\psi_{h} \in U_{h}$

$$
\left\|u-u_{h}\right\|_{U} \leq\left\|u-\psi_{h}\right\|_{U}+\left\|\psi_{h}-u_{h}\right\|_{U} \leq c\left\|u-\psi_{h}\right\|_{U} .
$$

Then the $\widetilde{H}^{1}(D)$-estimate follows from Theorem 3.2 and Lemma 4.2

$$
\left\|u-u_{h}\right\|_{U} \leq \inf _{\psi_{h} \in U_{h}} c\left\|u-\psi_{h}\right\|_{U} \leq c h^{\alpha-2+\beta}\|f\|_{L^{2}(D)},
$$

with $\beta \in(2-\alpha, 1 / 2)$. Next let $w$ be the solution of problem (3.7) with $F=u-u_{h}$. By Theorem 3.2 and Lemmas 4.2 and 4.6, we deduce

$$
\begin{aligned}
\left\|u-u_{h}\right\|_{L^{2}(D)}^{2} & =A\left(u-u_{h}, w\right) \leq c\left\|u-u_{h}\right\|_{U} \inf _{\varphi_{h} \in V_{h}}\left\|w-\varphi_{h}\right\|_{V} \\
& \leq c h^{\alpha-1+\beta}\|f\|_{L^{2}(D)}\left\|u-u_{h}\right\|_{L^{2}(D)} .
\end{aligned}
$$

Remark 4.2. Since the solution $u$ is in $\widetilde{H}^{1}(D) \cap H^{\alpha-1+\beta}(D)$ with $\beta \in(2-\alpha, 1 / 2)$, both $L^{2}(D)$ and $\widetilde{H}^{1}(D)$ error estimates are optimal. This is in stark contrast with that in [13], where the $L^{2}(D)$-error estimate suffers from one half order loss.

4.3. Error estimates in the Caputo case. Here the finite element problem reads: given any $F \in W^{*}$, find $u_{h} \in U_{h}$ such that

$$
A\left(u_{h}, \varphi_{h}\right)=\left\langle F, \varphi_{h}\right\rangle \quad \forall \varphi_{h} \in W_{h}
$$

First we prove the stability of problem (4.11) for the case $b, q \equiv 0$.

Lemma 4.7. Let $a(\cdot, \cdot)$ be the bilinear form in (3.10). Then there holds

$$
\sup _{\varphi_{h} \in W_{h}} \frac{a\left(\psi_{h}, \varphi_{h}\right)}{\left\|\varphi_{h}\right\|_{W}} \geq c\left\|\psi_{h}\right\|_{U} \quad \forall \psi_{h} \in U_{h},
$$

and the finite element problem: Find $u_{h} \in U_{h}$ such that

$$
a\left(u_{h}, \varphi_{h}\right)=\left(f, \varphi_{h}\right) \quad \forall \varphi_{h} \in W_{h},
$$

has a unique solution.

Proof. For any fixed $\psi_{h} \in U_{h}$, let $\varphi_{h}=-{ }_{x} I_{1}^{\alpha-1}\left(P_{W} \psi_{h}^{\prime}\right)$. Then $\varphi_{h} \in W_{h}$ and

$$
\begin{aligned}
a\left(\psi_{h}, \varphi_{h}\right) & =-\left(\psi_{h}^{\prime},{ }_{x}^{R} D_{1}^{\alpha-1}\left(-{ }_{x} I_{1}^{\alpha-1}\left(P_{W} \psi_{h}^{\prime}\right)\right)\right)=\left(\psi_{h}^{\prime}, P_{W} \psi_{h}^{\prime}\right) \\
& =\left(\psi_{h}^{\prime}, \psi_{h}^{\prime}\right)-c_{\alpha}\left({ }_{x} I_{1}^{\alpha-1} \psi_{h}^{\prime}, x^{1-\alpha}\right)\left(\psi_{h}^{\prime}, 1\right)=\left\|\psi_{h}\right\|_{U}^{2} .
\end{aligned}
$$


Further, the $L^{2}(D)$-stability of $P_{W}$ yields

$$
\left\|\varphi_{h}\right\|_{W}=\left\|_{x} I_{1}^{\alpha-1}\left(P_{W} \psi_{h}^{\prime}\right)\right\|_{W} \leq c\left\|P_{W} \psi_{h}^{\prime}\right\|_{L^{2}(D)} \leq c\left\|\psi_{h}\right\|_{U}
$$

Then we obtain (4.12) and the unique existence of a solution $u_{h} \in U_{h}$.

Next we introduce the (adjoint) Ritz projection $R_{h}: W \rightarrow W_{h}$ defined by

$$
a\left(\psi_{h}, R_{h} \varphi\right)=a\left(\psi_{h}, \varphi\right) \quad \forall \psi_{h} \in U_{h} .
$$

Analogous to Lemma 4.4, the following error estimates hold for $R_{h}$.

Lemma 4.8. The projection $R_{h}$ is well-defined and satisfies for any $\varphi \in W$

$$
\begin{aligned}
\left\|R_{h} \varphi\right\|_{W} & \leq c\|\varphi\|_{W}, \\
\left\|\varphi-R_{h} \varphi\right\|_{L^{2}(D)} & \leq c h^{\alpha-1}\|\varphi\|_{W} .
\end{aligned}
$$

Proof. For any given $\varphi_{h} \in W_{h}$, let $\psi_{h}={ }_{x} I_{1}^{2-\alpha} \varphi_{h}$. Clearly, $\psi_{h}(1)=0$ and $\psi_{h}(0)=$ $\left({ }_{x} I_{1}^{2-\alpha} \varphi_{h}\right)(0)=c_{\alpha}\left(x^{1-\alpha}, \varphi_{h}\right)=0$ since $\varphi_{h} \in W_{h} \subset W$. Consequently

$$
\left\|\varphi_{h}\right\|_{W} \leq c\left\|_{x}^{R} D_{1}^{\alpha-1} \varphi_{h}\right\|_{L^{2}(D)}=c\left\|\psi_{h}\right\|_{U}
$$

The discrete inf-sup condition follows from this and (3.12):

$$
\sup _{\psi_{h} \in U_{h}} \frac{a\left(\psi_{h}, \varphi_{h}\right)}{\left\|\psi_{h}\right\|_{U}} \geq c\left\|\varphi_{h}\right\|_{W} \quad \forall \varphi_{h} \in W_{h} .
$$

The $\widetilde{H}_{R}^{\alpha-1}(D)$-stability of $R_{h}$ follows immediately

$$
\left\|R_{h} \varphi\right\|_{W} \leq c \sup _{\psi_{h} \in U_{h}} \frac{a\left(\psi_{h}, R_{h} \varphi\right)}{\left\|\psi_{h}\right\|_{U}}=c \sup _{\psi_{h} \in U_{h}} \frac{a\left(\psi_{h}, \varphi\right)}{\left\|\psi_{h}\right\|_{U}} \leq c\|\varphi\|_{W} .
$$

Next let $g$ be the solution to (3.13) with $F=\varphi-R_{h} \varphi$. By Theorem 3.5, $\|g\|_{H^{\alpha}(D)} \leq$ $c\left\|\varphi-R_{h} \varphi\right\|_{L^{2}(D)}$. Then by the Galerkin orthogonality, Lemmas 4.8 and 4.2 , we deduce

$$
\begin{aligned}
\left\|\varphi-R_{h} \varphi\right\|_{L^{2}(D)}^{2} & =a\left(g, \varphi-R_{h} \varphi\right) \leq c \inf _{\psi_{h} \in U_{h}}\left\|g-\psi_{h}\right\|_{U}\left\|\varphi-R_{h} \varphi\right\|_{W} \\
& \leq c h^{\alpha-1}\left\|\varphi-R_{h} \varphi\right\|_{L^{2}(D)}\|\varphi\|_{W} .
\end{aligned}
$$

Remark 4.3. The $L^{2}(D)$ error estimate of $R_{h}$ in the Caputo case is optimal, since its adjoint problem has full regularity pickup.

Lemma 4.9. Let Assumption 3.2 hold, $f \in L^{2}(D)$, and $b, q \in L^{\infty}(D)$. Then there exists an $h_{0}$ such that for all $h \leq h_{0}$

$$
c\left\|\psi_{h}\right\|_{U} \leq \sup _{\varphi_{h} \in W_{h}} \frac{A\left(\psi_{h}, \varphi_{h}\right)}{\left\|\varphi_{h}\right\|_{W}} \quad \forall \psi_{h} \in U_{h} .
$$

For such $h$, the finite element problem: Find $u_{h} \in U_{h}$ such that

$$
A\left(u_{h}, \varphi_{h}\right)=\left(f, \varphi_{h}\right) \quad \forall \varphi_{h} \in W_{h},
$$

has a unique solution.

Proof. The proof is the same as that of Lemma 4.5, using Lemmas 4.7 and 4.8.

Lemma 4.10. Let Assumption 3.2 hold, $f \in L^{2}(D), b \in W^{1, \infty}(D)$ and $q \in L^{\infty}(D)$. Let $w$ be the solution of problem (3.14). Then there holds

$$
\inf _{\psi_{h} \in W_{h}}\left\|w-\psi_{h}\right\|_{L^{2}(D)}+\inf _{\psi_{h} \in W_{h}}\left\|w-\psi_{h}\right\|_{W} \leq \operatorname{ch}\|f\|_{L^{2}(D)} .
$$


Proof. The proof is identical with that of Lemma 4.6, with $P_{W}$ in place of $P_{V}$.

Theorem 4.2. Let $s \in[0,1 / 2)$ and Assumption 3.2 hold. Suppose $f \in H^{s}(D)$, $b \in W^{1, \infty}(D)$ and $q \in L^{\infty}(D) \cap H^{s}(D)$. Then there exists an $h_{0}$ such that for all $h \leq h_{0}$, the solution $u_{h}$ to the finite element problem (4.14) satisfies

$$
\left\|u-u_{h}\right\|_{L^{2}(D)}+h\left\|u-u_{h}\right\|_{U} \leq c h^{\min (\alpha+s, 2)}\|f\|_{H^{s}(D)} .
$$

Proof. The $H^{1}(D)$-estimate follows directly from Cea's lemma and Lemma 4.10 as in Theorem 4.1. With $w$ being the solution to problem (3.14) with $F=u-u_{h}$, by Theorem 3.5, Lemmas 4.2 and 4.10, we deduce

$$
\begin{aligned}
\left\|u-u_{h}\right\|_{L^{2}(D)}^{2} & =A\left(u-u_{h}, w\right) \leq c\left\|u-u_{h}\right\|_{U} \inf _{\varphi_{h} \in W_{h}}\left\|w-\varphi_{h}\right\|_{W} \\
& \leq c h^{\min (\alpha+s, 2)}\|f\|_{H^{s}(D)}\left\|u-u_{h}\right\|_{L^{2}(D)} .
\end{aligned}
$$

4.4. Numerical implementation. Now we briefly discuss the efficient implementation of the Petrov-Galerkin FEM on a uniform mesh, especially the computation of the stiffness matrix $\mathbf{S}=\mathbf{A}+\mathbf{R}:=\left[a_{i, j}\right]+\left[r_{i, j}\right]$, with

$$
a_{i, j}=-\left(\psi_{j}^{\prime},{ }_{x}^{R} D_{1}^{\alpha-1} \varphi_{i}\right) \text { and } r_{i, j}=\left(b \psi_{j}^{\prime}, \varphi_{i}\right)+\left(q \psi_{j}, \varphi_{i}\right),
$$

where $\psi_{j}$ and $\varphi_{i}$ are the basis functions in $U_{h}$ and in $V_{h}$ or $W_{h}$, respectively. In the space $U_{h}$, we choose the nodal basis function $\left\{\psi_{j}\right\}$ :

$$
\psi_{j}=\left\{\begin{array}{rc}
\left(x-x_{j-1}\right) / h & \text { for } x \in\left[x_{j-1}, x_{j}\right), \\
\left(x_{j+1}-x\right) / h & \text { for } x \in\left[x_{j}, x_{j+1}\right), \\
0 & \text { otherwise },
\end{array}\right.
$$

with $j=1,2, . ., m-1$. Now, we set the basis function $\varphi_{i}$ of $V_{h}$ by

$$
\varphi_{i}=\left(x_{i}-x\right)^{\alpha-1} \chi_{\left[0, x_{i}\right]}-x_{i}^{\alpha-1}(1-x)^{\alpha-1}, i=1,2, . ., m-1 .
$$

Clearly, $\varphi_{i} \in V_{h}$, and ${ }_{x}^{R} D_{1}^{\alpha-1} \varphi_{i}$ is piecewise constant $-{ }_{x}^{R} D_{1}^{\alpha-1} \varphi_{i}=-\Gamma(\alpha) \chi_{\left[0, x_{i}\right]}+$ $x_{i}^{\alpha-1} \Gamma(\alpha)$. Hence we have

$$
a_{i, j}=-\left(\psi_{j}^{\prime},{ }_{x}^{R} D_{1}^{\alpha-1} \varphi_{i}\right)=-\Gamma(\alpha) \delta_{i j} / h,
$$

where $\delta_{i j}$ is the Kronecker symbol. That is, the matrix $\mathbf{A}$ is a multiple of the identity matrix, which is one distinct feature of the proposed approach. Hence, the resulting linear system is well conditioned, and $\mathbf{A}$ can be used as a preconditioner, if desired. The matrix $\mathbf{R}$ can be accurately computed using quadrature rules.

Likewise, we define the basis function $\varphi_{i}$ in $W_{h}$ by

$$
\varphi_{i}=\left(x_{i}-x\right)^{\alpha-1} \chi_{\left[0, x_{i}\right]}-x_{i}(1-x)^{\alpha-1} \quad \text { with } i=1,2, . ., m-1 .
$$

By $\left(\varphi_{i}, x^{1-\alpha}\right)=0, \varphi_{i} \in W_{h}$, and thus (4.15) holds also in the Caputo case.

\section{An enriched FEM in the Riemann-Liouville Case}

By Theorem 4.1, in the Riemann-Liouville case the FEM can only converge slowly, due to the presence of the singular term $x^{\alpha-1}$. Now we discuss how to improve the convergence, using an idea first introduced in [4] for the Poisson equation on an L-shaped domain, and then extended to FBVPs in [15]. Below we only sketch the technique and state the result, since the proofs are analogous to [15]. 
This technique is to split the solution $u$ to problem (1.1) into a regular part $u^{r}$ and a singular part involving $x^{\alpha-1}$ (with $\left.u^{s}=x^{\alpha-1}-x^{2}\right)$ :

$$
u(x)=u^{r}+\mu u^{s} .
$$

We shall assume ${ }_{0} I_{x}^{\alpha}\left(b\left(u^{s}\right)^{\prime}+q u^{s}\right)(1) \neq-1$. Otherwise, we may replace the choice $x^{2}$ by any other function $v$ in the space $\widetilde{H}_{L}^{s}(D), s \geq 2$, with $v(1)=1$, such that ${ }_{0} I_{x}^{\alpha}\left(b\left(x^{\alpha-1}-v\right)^{\prime}+q\left(x^{\alpha-1}-v\right)\right)(1) \neq-1$. Then the regular part $u^{r}$ is given by

$$
u^{r}=-{ }_{0} I_{x}^{\alpha}\left(f-b u^{\prime}-q u\right)+\left({ }_{0} I_{x}^{\alpha}\left(f-b u^{\prime}-q u\right)\right)(1) x^{2},
$$

and the singularity strength $\mu$ is given by $\mu=c_{0}\left({ }_{0} I_{x}^{\alpha}\left(f-b\left(u^{r}\right)^{\prime}-q u^{r}\right)\right)(1)$, where $c_{0}=1 /\left(1+{ }_{0} I_{x}^{\alpha}\left(b\left(u^{s}\right)^{\prime}+q u^{s}\right)(1)\right)$. The regular part $u^{r}$ satisfies

$$
-{ }_{0}^{R} D_{x}^{\alpha} u^{r}+b\left(u^{r}\right)^{\prime}+q u^{r}+\left({ }_{0} I_{x}^{\alpha}\left(b\left(u^{r}\right)^{\prime}+q u^{r}\right)\right)(1) Q=\tilde{f} \quad \text { in } D,
$$

with $u^{r}(0)=u^{r}(1)=0$, where the functions $Q$ and $\tilde{f}$ are defined by $Q=c_{0}^{R} D_{x}^{\alpha} u^{s}-$ $c_{0} b\left(u^{s}\right)^{\prime}-c_{0} q u^{s} \in L^{2}(D)$ and $\tilde{f}=f+c_{0}\left({ }_{0} I_{x}^{\alpha} f\right)(1)\left({ }_{0}^{R} D_{x}^{\alpha} u^{s}-b\left(u^{s}\right)^{\prime}-q u^{s}\right) \in L^{2}(D)$, respectively. Now we introduce a bilinear form $A_{r}(\cdot, \cdot): U \times V \rightarrow \mathbb{R}$ by

$$
A_{r}(u, \varphi)=a(u, \varphi)+b(u, \varphi),
$$

with $b(u, \varphi)=\left(b u^{\prime}+q u, \varphi\right)+{ }_{0} I_{x}^{\alpha}\left(b u^{\prime}+q u\right)(1)(Q, \varphi)$. Then the variational formulation of the regular part $u^{r}$ is to find $u^{r} \in U$ such that

$$
A_{r}\left(u^{r}, \varphi\right)=(\widetilde{f}, \varphi) \quad \forall \varphi \in V .
$$

The following assumption on $A_{r}(\cdot, \cdot)$ is analogous to Assumption 3.1.

Assumption 5.1. Let the bilinear form $A_{r}(\cdot, \cdot): U \times V \rightarrow \mathbb{R}$ satisfy

(a) The problem of finding $u \in U$ such that $A_{r}(u, \varphi)=0$ for all $\varphi \in V$ has only the trivial solution $u \equiv 0$.

$\left(\mathrm{a}^{*}\right)$ The problem of finding $\varphi \in V$ such that $A_{r}(u, \varphi)=0$ for all $u \in U$ has only the trivial solution $\varphi \equiv 0$.

Under Assumption 5.1, problem (5.2) is stable and has extra regularity pickup.

Theorem 5.1. Let Assumption 5.1 hold, $b, q \in \widetilde{H}^{\gamma}(D) \cap L^{\infty}(D)$ and $f \in \widetilde{H}_{L}^{\gamma}(D)$ with $\gamma>\alpha-3 / 2$. Then there exists a unique solution $u^{r} \in H^{2 \alpha-2+\beta}(D) \cap \widetilde{H}^{1}(D)$, $\beta \in[2-\alpha, 1 / 2)$, to problem (5.2) and further, it satisfies

$$
\left\|u^{r}\right\|_{H^{2 \alpha-2+\beta}(D)} \leq c\|f\|_{\tilde{H}_{L}^{\gamma}(D)} .
$$

Proof. The proof of the stability is similar to that in Section 3.1. The regularity estimate follows from the representation (5.1) and Theorem 3.2.

Now we consider the discrete problem: find $u_{h}^{r} \in U_{h}$ such that

$$
A_{r}\left(u_{h}^{r}, \varphi_{h}\right)=\left(\tilde{f}, \varphi_{h}\right) \quad \forall \varphi_{h} \in V_{h} .
$$

Then we reconstruct $u_{h}$ by

$$
u_{h}=u_{h}^{r}+\mu_{h} u^{s} \quad \text { with } \quad \mu_{h}=c_{0}\left({ }_{0} I_{x}^{\alpha}\left(f-b u_{h}^{r \prime}-q u_{h}^{r}\right)\right)(1) .
$$

Last, we state error estimates of the approximation $u_{h}$.

Theorem 5.2. Let Assumption 5.1 hold, $b \in W^{1, \infty}(D), q \in H^{1}(D) \cap L^{\infty}(D)$ and $f \in \widetilde{H}_{L}^{\gamma}(D), \gamma>\alpha-3 / 2$. Then there exists an $h_{0}$ such that for all $h \leq h_{0}$, the solution $u_{h}$ to problem (5.3)-(5.4) satisfies for any $\beta \in(2-\alpha, 1 / 2)$,

$$
\left\|u-u_{h}\right\|_{L^{2}(D)}+h\left\|u-u_{h}\right\|_{U} \leq c h^{\min (2 \alpha-2+\beta, 2)}\|f\|_{\widetilde{H}_{L}^{\gamma}(D)} .
$$




\section{NumERICAL RESULTS AND DISCUSSIONS}

Now we present numerical experiments to verify the convergence theory, and consider the following three examples:

(a) The source term $f=x \in \widetilde{H}_{L}^{s}(D), s \in(1,3 / 2)$.

(b) The source term $f=1 \in \widetilde{H}_{L}^{s}(D), s \in(0,1 / 2)$.

(c) The source term $f=x^{-1 / 4} \in \widetilde{H}_{L}^{s}(D), s \in(0,1 / 4)$.

The numerical results are computed on a uniform mesh with a mesh size $h=1 / \mathrm{m}$, $m \in \mathbb{N}$. All the numerical experiments are performed on a personal computer with MATLAB 2014a. In the case of $q, b \equiv 0$, the exact solution is available in closed form, cf. (3.1) and (3.9). In general, the analytic solution is not available, and a reference solution is computed on a much finer mesh with a mesh size $h=1 / 5000$.

6.1. Numerical results for example (a). The numerical results for case (a) with $b, q \equiv 0$ are given in Tables 1 and 2 for the Riemann-Liouville and Caputo derivative, respectively. The notation rate in the tables refers to empirical convergence rate, and the numbers in the bracket denote the theoretical predictions from Section 4. The empirical rates agree well with the theoretical ones for all three fractional orders. As the order $\alpha$ increases, the convergence rate in the $L^{2}(D)$ and $\widetilde{H}^{1}(D)$ norm improves accordingly. In the Riemann-Liouville case, despite the smoothness of the source term $f$, the solution regularity is limited, due to the presence of the singularity $x^{\alpha-1}$. These observations remain valid for the Caputo derivative, but the convergence rates are higher. The estimates in Section 4 are sharp for both derivatives. Further, we have the following interesting observation: for $i=$ $1,2, \ldots, m-1$

$$
\Gamma(\alpha) u\left(x_{i}\right)=\left(u^{\prime},{ }_{x}^{R} D_{1}^{\alpha-1} \psi_{i}\right)=-\left(f, \psi_{i}\right)=\left(u_{h}^{\prime},{ }_{x}^{R} D_{1}^{\alpha-1} \psi_{i}\right)=\Gamma(\alpha) u_{h}\left(x_{i}\right) .
$$

That is, the solution $u_{h}$ coincides with the $P 1$ Lagrange interpolation of $u$. This partly implies optimality of the convergence rates in Section 4 . The presence of a smooth $b$ and $q$ does not affect the convergence rates, cf. Tables 3 and 4 .

TABle 1. Numerical results for example (a) with a RiemannLiouville derivative and $b, q=0, \alpha=1.6,1.75,1.9, h=1 / m$.

\begin{tabular}{|c|c|cccccc|c|}
\hline$\alpha$ & $m$ & 10 & 20 & 40 & 80 & 160 & 320 & rate \\
\hline 1.6 & $L^{2}$ & $3.10 \mathrm{e}-3$ & $1.39 \mathrm{e}-3$ & $6.42 \mathrm{e}-4$ & $2.99 \mathrm{e}-4$ & $1.39 \mathrm{e}-4$ & $6.47 \mathrm{e}-5$ & $\approx 1.10(1.10)$ \\
& $H^{1}$ & $1.67 \mathrm{e}-1$ & $1.50 \mathrm{e}-1$ & $1.35 \mathrm{e}-1$ & $1.21 \mathrm{e}-1$ & $1.07 \mathrm{e}-1$ & $9.33 \mathrm{e}-2$ & $\approx 0.17(0.10)$ \\
\hline 1.75 & $L^{2}$ & $1.25 \mathrm{e}-3$ & $4.62 \mathrm{e}-4$ & $1.84 \mathrm{e}-4$ & $7.55 \mathrm{e}-5$ & $3.15 \mathrm{e}-5$ & $1.32 \mathrm{e}-5$ & $\approx 1.27(1.25)$ \\
& $H^{1}$ & $5.03 \mathrm{e}-2$ & $3.89 \mathrm{e}-2$ & $3.14 \mathrm{e}-2$ & $2.57 \mathrm{e}-2$ & $2.10 \mathrm{e}-2$ & $1.70 \mathrm{e}-2$ & $\approx 0.29(0.25)$ \\
\hline 1.9 & $L^{2}$ & $6.40 \mathrm{e}-4$ & $1.72 \mathrm{e}-4$ & $4.92 \mathrm{e}-5$ & $1.53 \mathrm{e}-5$ & $5.14 \mathrm{e}-6$ & $1.83 \mathrm{e}-6$ & $\approx 1.53(1.40)$ \\
& $H^{1}$ & $2.08 \mathrm{e}-2$ & $1.15 \mathrm{e}-2$ & $6.81 \mathrm{e}-3$ & $4.38 \mathrm{e}-3$ & $3.01 \mathrm{e}-3$ & $2.14 \mathrm{e}-3$ & $\approx 0.50(0.40)$ \\
\hline
\end{tabular}

One distinct feature of the proposed approach is that the stiffness matrix for the leading term is diagonal, and the resulting linear system is well conditioned. To illustrate this, we give in Table 5 the condition numbers of the stiffness matrix for $\alpha=1.55,1.75$ and 1.95 . It is observed that for either derivative, it is fairly small for the whole range of fractional orders, and independent of the mesh size $h$. These results fully confirm the observations in Section 4.4. 
A PETROV-GALERKIN FINITE ELEMENT METHOD FOR FRACTIONAL CONVECTION-DIFFUSION EQUATIONg

TABLe 2. Numerical results for example (a) with a Caputo derivative and $b, q=0, \alpha=1.6,1.75,1.9, h=1 / m$.

\begin{tabular}{|c|c|cccccc|c|}
\hline$\alpha$ & $m$ & 10 & 20 & 40 & 80 & 160 & 320 & rate \\
\hline 1.6 & $L^{2}$ & $6.88 \mathrm{e}-4$ & $1.72 \mathrm{e}-4$ & $4.30 \mathrm{e}-5$ & $1.08 \mathrm{e}-5$ & $2.69 \mathrm{e}-6$ & $6.71 \mathrm{e}-7$ & $\approx 2.00(2.00)$ \\
& $H^{1}$ & $2.18 \mathrm{e}-2$ & $1.09 \mathrm{e}-2$ & $5.45 \mathrm{e}-3$ & $2.72 \mathrm{e}-3$ & $1.33 \mathrm{e}-3$ & $6.47 \mathrm{e}-4$ & $\approx 1.02(1.00)$ \\
\hline 1.75 & $L^{2}$ & $6.28 \mathrm{e}-4$ & $1.57 \mathrm{e}-4$ & $3.93 \mathrm{e}-5$ & $9.81 \mathrm{e}-6$ & $2.45 \mathrm{e}-6$ & $6.12 \mathrm{e}-7$ & $\approx 2.00(2.00)$ \\
& $H^{1}$ & $1.99 \mathrm{e}-2$ & $9.93 \mathrm{e}-3$ & $4.97 \mathrm{e}-3$ & $2.48 \mathrm{e}-3$ & $1.22 \mathrm{e}-3$ & $5.90 \mathrm{e}-4$ & $\approx 1.02(1.00)$ \\
\hline 1.9 & $L^{2}$ & $5.67 \mathrm{e}-4$ & $1.42 \mathrm{e}-4$ & $3.54 \mathrm{e}-5$ & $8.86 \mathrm{e}-6$ & $2.21 \mathrm{e}-6$ & $5.53 \mathrm{e}-7$ & $\approx 2.00(2.00)$ \\
& $H^{1}$ & $1.79 \mathrm{e}-2$ & $8.97 \mathrm{e}-3$ & $4.48 \mathrm{e}-3$ & $2.24 \mathrm{e}-3$ & $1.10 \mathrm{e}-3$ & $5.33 \mathrm{e}-4$ & $\approx 1.02(1.00)$ \\
\hline
\end{tabular}

TABLE 3. Numerical results for example (a) with a RiemannLiouville derivative and $b=e^{x}, q=x(1-x), \alpha=1.6,1.75,1.9$, $h=1 / m$.

\begin{tabular}{|c|c|cccccc|c|}
\hline$\alpha$ & $m$ & 10 & 20 & 40 & 80 & 160 & 320 & rate \\
\hline 1.6 & $L^{2}$ & $2.67 \mathrm{e}-3$ & $9.41 \mathrm{e}-4$ & $3.89 \mathrm{e}-4$ & $1.74 \mathrm{e}-4$ & $8.01 \mathrm{e}-5$ & $3.69 \mathrm{e}-5$ & $\approx 1.13(1.10)$ \\
& $H^{1}$ & $1.22 \mathrm{e}-1$ & $9.14 \mathrm{e}-2$ & $7.93 \mathrm{e}-2$ & $6.65 \mathrm{e}-2$ & $5.81 \mathrm{e}-2$ & $5.00 \mathrm{e}-2$ & $\approx 0.20(0.10)$ \\
\hline 1.75 & $L^{2}$ & $1.23 \mathrm{e}-3$ & $3.69 \mathrm{e}-4$ & $1.28 \mathrm{e}-4$ & $4.92 \mathrm{e}-5$ & $2.00 \mathrm{e}-5$ & $8.29 \mathrm{e}-6$ & $\approx 1.29(1.25)$ \\
& $H^{1}$ & $5.25 \mathrm{e}-2$ & $3.18 \mathrm{e}-2$ & $2.18 \mathrm{e}-2$ & $1.65 \mathrm{e}-2$ & $1.31 \mathrm{e}-2$ & $1.04 \mathrm{e}-2$ & $\approx 0.33(0.25)$ \\
\hline 1.9 & $L^{2}$ & $7.49 \mathrm{e}-4$ & $1.92 \mathrm{e}-4$ & $5.05 \mathrm{e}-5$ & $1.40 \mathrm{e}-5$ & $4.20 \mathrm{e}-6$ & $1.37 \mathrm{e}-6$ & $\approx 1.66(1.40)$ \\
& $H^{1}$ & $3.02 \mathrm{e}-2$ & $1.55 \mathrm{e}-2$ & $8.10 \mathrm{e}-3$ & $4.44 \mathrm{e}-3$ & $2.61 \mathrm{e}-3$ & $1.65 \mathrm{e}-3$ & $\approx 0.70(0.40)$ \\
\hline
\end{tabular}

TABle 4. Numerical results for example (a) with a Caputo derivative and $b=e^{x}, q=x(1-x), \alpha=1.6,1.75,1.9, h=1 / m$.

\begin{tabular}{|c|c|cccccc|c|}
\hline$\alpha$ & $m$ & 10 & 20 & 40 & 80 & 160 & 320 & rate \\
\hline 1.6 & $L^{2}$ & $1.91 \mathrm{e}-3$ & $4.92 \mathrm{e}-4$ & $1.25 \mathrm{e}-4$ & $3.18 \mathrm{e}-5$ & $8.03 \mathrm{e}-6$ & $2.01 \mathrm{e}-6$ & $\approx 1.99(2.00)$ \\
& $H^{1}$ & $7.12 \mathrm{e}-2$ & $3.59 \mathrm{e}-2$ & $1.80 \mathrm{e}-2$ & $9.00 \mathrm{e}-3$ & $4.50 \mathrm{e}-3$ & $2.12 \mathrm{e}-3$ & $\approx 1.03(1.00)$ \\
\hline 1.75 & $L^{2}$ & $1.03 \mathrm{e}-3$ & $2.59 \mathrm{e}-4$ & $6.49 \mathrm{e}-5$ & $1.62 \mathrm{e}-5$ & $4.06 \mathrm{e}-6$ & $1.01 \mathrm{e}-6$ & $\approx 2.00(2.00)$ \\
& $H^{1}$ & $4.18 \mathrm{e}-2$ & $2.10 \mathrm{e}-2$ & $1.05 \mathrm{e}-2$ & $5.27 \mathrm{e}-3$ & $2.63 \mathrm{e}-3$ & $1.24 \mathrm{e}-3$ & $\approx 1.00(1.00)$ \\
\hline 1.9 & $L^{2}$ & $7.22 \mathrm{e}-4$ & $1.81 \mathrm{e}-4$ & $4.53 \mathrm{e}-5$ & $1.13 \mathrm{e}-5$ & $2.83 \mathrm{e}-6$ & $7.04 \mathrm{e}-7$ & $\approx 2.00(2.00)$ \\
& $H^{1}$ & $2.88 \mathrm{e}-2$ & $1.45 \mathrm{e}-2$ & $7.25 \mathrm{e}-3$ & $3.62 \mathrm{e}-3$ & $1.81 \mathrm{e}-3$ & $8.52 \mathrm{e}-4$ & $\approx 1.02(1.00)$ \\
\hline
\end{tabular}

TABLE 5. The condition number of the linear system for $b(x)=e^{x}$, $q(x)=x(1-x), \alpha=1.55,1.75$ and $1.95, h=1 / m$.

\begin{tabular}{|c|c|ccccccc|}
\hline Deriv. type & $\alpha \backslash m$ & 20 & 40 & 80 & 160 & 320 & 640 & 1280 \\
\hline \multirow{3}{*}{ R-L } & 1.55 & 2.98 & 3.48 & 4.26 & 4.30 & 4.57 & 4.84 & 5.00 \\
& 1.75 & 2.06 & 2.22 & 2.33 & 2.40 & 2.45 & 2.48 & 2.50 \\
& 1.95 & 1.63 & 1.68 & 1.71 & 1.73 & 1.74 & 1.74 & 1.75 \\
\hline \multirow{3}{*}{ Caputo } & 1.55 & 2.75 & 3.20 & 3.57 & 3.89 & 4.16 & 4.39 & 4.60 \\
& 1.75 & 2.02 & 2.17 & 2.27 & 2.34 & 2.39 & 2.42 & 2.44 \\
& 1.95 & 1.63 & 1.68 & 1.71 & 1.73 & 1.73 & 1.74 & 1.74 \\
\hline
\end{tabular}

6.2. Numerical results for example (b). Here the source term $f$ is smooth but does not satisfy the zero boundary condition. In the Riemann-Liouville case, the 
$L^{2}(D)$ and $H^{1}(D)$ errors are respectively of order $O\left(h^{\alpha-1 / 2}\right)$ and $O\left(h^{\alpha-3 / 2}\right)$, while in the Caputo case, an $O(h)$ and $O\left(h^{2}\right)$ rate is observed for $L^{2}(D)$ and $H^{1}(D)$ errors, respectively, cf. Tables 6 and 7 , which fully confirm our convergence theory.

TABLe 6. Numerical results for example (b) with a RiemannLiouville derivative and $b=e^{x}, q=x(1-x), \alpha=1.6,1.75,1.9$, $h=1 / m$.

\begin{tabular}{|c|c|cccccc|c|}
\hline$\alpha$ & $m$ & 10 & 20 & 40 & 80 & 160 & 320 & rate \\
\hline 1.6 & $L^{2}$ & $6.87 \mathrm{e}-3$ & $2.89 \mathrm{e}-3$ & $1.28 \mathrm{e}-3$ & $5.78 \mathrm{e}-4$ & $2.65 \mathrm{e}-4$ & $1.22 \mathrm{e}-4$ & $\approx 1.12(1.10)$ \\
& $H^{1}$ & $3.28 \mathrm{e}-1$ & $2.79 \mathrm{e}-1$ & $2.46 \mathrm{e}-1$ & $2.17 \mathrm{e}-1$ & $1.90 \mathrm{e}-1$ & $1.63 \mathrm{e}-1$ & $\approx 0.20(0.10)$ \\
\hline 1.75 & $L^{2}$ & $2.93 \mathrm{e}-3$ & $1.05 \mathrm{e}-3$ & $4.04 \mathrm{e}-4$ & $1.62 \mathrm{e}-4$ & $6.65 \mathrm{e}-5$ & $2.76 \mathrm{e}-5$ & $\approx 1.28(1.25)$ \\
& $H^{1}$ & $1.17 \mathrm{e}-1$ & $8.47 \mathrm{e}-2$ & $6.59 \mathrm{e}-2$ & $5.29 \mathrm{e}-2$ & $4.28 \mathrm{e}-2$ & $3.42 \mathrm{e}-2$ & $\approx 0.31(0.25)$ \\
\hline 1.9 & $L^{2}$ & $1.37 \mathrm{e}-3$ & $3.82 \mathrm{e}-4$ & $1.12 \mathrm{e}-4$ & $3.54 \mathrm{e}-5$ & $1.19 \mathrm{e}-5$ & $4.23 \mathrm{e}-6$ & $\approx 1.53(1.40)$ \\
& $H^{1}$ & $5.16 \mathrm{e}-2$ & $2.84 \mathrm{e}-2$ & $1.66 \mathrm{e}-2$ & $1.04 \mathrm{e}-2$ & $7.02 \mathrm{e}-3$ & $4.91 \mathrm{e}-3$ & $\approx 0.54(0.40)$ \\
\hline
\end{tabular}

TABle 7. Numerical results for example (b) with a Caputo derivative and $b=e^{x}, q=x(1-x), \alpha=1.6,1.75,1.9, h=1 / m$.

\begin{tabular}{|c|c|cccccc|c|}
\hline$\alpha$ & $m$ & 10 & 20 & 40 & 80 & 160 & 320 & rate \\
\hline 1.6 & $L^{2}$ & $1.86 \mathrm{e}-3$ & $4.72 \mathrm{e}-4$ & $1.19 \mathrm{e}-4$ & $3.00 \mathrm{e}-5$ & $7.54 \mathrm{e}-6$ & $1.88 \mathrm{e}-6$ & $\approx 2.00(2.00)$ \\
& $H^{1}$ & $7.89 \mathrm{e}-2$ & $3.98 \mathrm{e}-2$ & $2.00 \mathrm{e}-2$ & $1.00 \mathrm{e}-2$ & $5.00 \mathrm{e}-3$ & $2.35 \mathrm{e}-3$ & $\approx 1.03(1.00)$ \\
\hline 1.75 & $L^{2}$ & $1.28 \mathrm{e}-3$ & $3.22 \mathrm{e}-4$ & $8.03 \mathrm{e}-5$ & $2.01 \mathrm{e}-5$ & $5.01 \mathrm{e}-6$ & $1.25 \mathrm{e}-6$ & $\approx 2.00(2.00)$ \\
& $H^{1}$ & $5.38 \mathrm{e}-2$ & $2.71 \mathrm{e}-2$ & $1.35 \mathrm{e}-2$ & $6.78 \mathrm{e}-3$ & $3.39 \mathrm{e}-3$ & $1.59 \mathrm{e}-3$ & $\approx 1.03(1.00)$ \\
\hline 1.9 & $L^{2}$ & $1.07 \mathrm{e}-3$ & $2.68 \mathrm{e}-4$ & $6.71 \mathrm{e}-5$ & $1.68 \mathrm{e}-5$ & $4.19 \mathrm{e}-6$ & $1.04 \mathrm{e}-6$ & $\approx 2.00(2.00)$ \\
& $H^{1}$ & $4.20 \mathrm{e}-2$ & $2.11 \mathrm{e}-2$ & $1.05 \mathrm{e}-2$ & $5.28 \mathrm{e}-3$ & $2.64 \mathrm{e}-3$ & $1.24 \mathrm{e}-3$ & $\approx 1.04(1.00)$ \\
\hline
\end{tabular}

6.3. Numerical results for example (c). Note that the source term $f(x)=$ $x^{-1 / 4} \in \widetilde{H}^{s}(D)$ with $s \in[0,1 / 4)$. Hence, in the Caputo case with $\alpha<1.75$, the solution $u$ fails to be in $H^{2}(D)$, which deteriorates the convergence rate. The $H^{1}(D)$ and $L^{2}(D)$-errors are of order $O\left(h^{0.85}\right)$ and $O\left(h^{1.85}\right)$ in case of $\alpha=1.6$, while for $\alpha=1.75$ and 1.9, an $O(h)$ and $O\left(h^{2}\right)$ rate of the $H^{1}(D)$ and $L^{2}(D)$-errors is observed, cf. Table 9, confirming theoretical predictions. In the Riemann-Liouville case, the desired optimal but slow convergence behavior is observed, cf. Table 8 .

6.4. Numerical results for the enriched FEM. In Table 10, we present the $L^{2}(D)$ and $H^{1}(D)$ norms of the error in approximating the regular part $u^{r}$ for example (b). Since $f \in \widetilde{H}^{s}(D)$ with $s \in[0,1 / 2)$, by Theorem 5.1, $u^{r} \in H^{2}(D)$ in case of $\alpha>1.75$. The numerical results show a convergence rate of $O\left(h^{2}\right)$ and $O(h)$ for the $L^{2}(D)$ and $H^{1}(D)$-norms of the error, respectively, for $\alpha=1.75$ and 1.9. For $\alpha=1.6$, the regular part $u^{r}$ lies in $H^{1.2+\beta}(D)$ with $\beta \in[2-\alpha, 1 / 2)$ by Theorem 5.1, and we observe a convergence rate $O\left(h^{1.7}\right)$ and $O\left(h^{0.7}\right)$, respectively, in $L^{2}(D)$ and $H^{1}(D)$-norm, which fully confirms Theorem 5.2. The error $\left|\mu-\mu_{h}\right|$ of the reconstructed singular strength $\mu_{h}$ achieves an $O\left(h^{2}\right)$ convergence, even for $\alpha=1.6$, cf. Table 11 . 
TABLe 8. Numerical results for example (c) with the RiemannLiouville derivative and $b=e^{x}, q=x(1-x), \alpha=1.6,1.75,1.9$, $h=1 / m$.

\begin{tabular}{|c|c|cccccc|c|}
\hline$\alpha$ & $m$ & 10 & 20 & 40 & 80 & 160 & 320 & rate \\
\hline 1.6 & $L^{2}$ & $1.07-2$ & $4.61 \mathrm{e}-3$ & $2.04 \mathrm{e}-3$ & $9.18 \mathrm{e}-4$ & $4.18 \mathrm{e}-4$ & $1.91 \mathrm{e}-4$ & $\approx 1.13(1.10)$ \\
& $H^{1}$ & $5.03 \mathrm{e}-1$ & $4.38 \mathrm{e}-1$ & $3.87 \mathrm{e}-1$ & $3.42 \mathrm{e}-1$ & $2.99 \mathrm{e}-1$ & $2.57 \mathrm{e}-1$ & $\approx 0.20(0.10)$ \\
\hline 1.75 & $L^{2}$ & $4.62 \mathrm{e}-3$ & $1.71 \mathrm{e}-3$ & $6.60 \mathrm{e}-4$ & $2.63 \mathrm{e}-4$ & $1.07 \mathrm{e}-4$ & $4.40 \mathrm{e}-5$ & $\approx 1.30(1.25)$ \\
& $H^{1}$ & $1.76 \mathrm{e}-1$ & $1.33 \mathrm{e}-1$ & $1.05 \mathrm{e}-1$ & $8.47 \mathrm{e}-2$ & $6.83 \mathrm{e}-2$ & $5.44 \mathrm{e}-2$ & $\approx 0.32(0.25)$ \\
\hline 1.9 & $L^{2}$ & $2.02 \mathrm{e}-3$ & $5.93 \mathrm{e}-4$ & $1.82 \mathrm{e}-4$ & $5.87 \mathrm{e}-5$ & $1.99 \mathrm{e}-5$ & $6.99 \mathrm{e}-6$ & $\approx 1.53(1.40)$ \\
& $H^{1}$ & $7.05 \mathrm{e}-2$ & $4.12 \mathrm{e}-2$ & $2.54 \mathrm{e}-2$ & $1.66 \mathrm{e}-2$ & $1.14 \mathrm{e}-2$ & $7.97 \mathrm{e}-3$ & $\approx 0.52(0.40)$ \\
\hline
\end{tabular}

TABle 9. Numerical results for example (c) with a Caputo derivative and $b=e^{x}, q=x(1-x), \alpha=1.6,1.75,1.9, h=1 / m$.

\begin{tabular}{|c|c|cccccc|c|}
\hline$\alpha$ & $m$ & 10 & 20 & 40 & 80 & 160 & 320 & rate \\
\hline 1.6 & $L^{2}$ & $1.84 \mathrm{e}-3$ & $4.92 \mathrm{e}-4$ & $1.31 \mathrm{e}-4$ & $3.51 \mathrm{e}-5$ & $9.46 \mathrm{e}-6$ & $2.56 \mathrm{e}-6$ & $\approx 1.89(1.85)$ \\
& $H^{1}$ & $7.47 \mathrm{e}-2$ & $3.87 \mathrm{e}-2$ & $2.01 \mathrm{e}-2$ & $1.05 \mathrm{e}-2$ & $5.54 \mathrm{e}-3$ & $2.82 \mathrm{e}-3$ & $\approx 0.94(0.85)$ \\
\hline 1.75 & $L^{2}$ & $1.56 \mathrm{e}-3$ & $4.05 \mathrm{e}-4$ & $1.05 \mathrm{e}-4$ & $2.72 \mathrm{e}-5$ & $7.04 \mathrm{e}-6$ & $1.81 \mathrm{e}-6$ & $\approx 1.97(2.00)$ \\
& $H^{1}$ & $5.92 \mathrm{e}-2$ & $3.04 \mathrm{e}-2$ & $1.56 \mathrm{e}-2$ & $7.99 \mathrm{e}-3$ & $4.09 \mathrm{e}-3$ & $1.98 \mathrm{e}-3$ & $\approx 0.99(1.00)$ \\
\hline 1.9 & $L^{2}$ & $1.39 \mathrm{e}-3$ & $3.54 \mathrm{e}-4$ & $8.99 \mathrm{e}-5$ & $2.28 \mathrm{e}-5$ & $5.74 \mathrm{e}-6$ & $1.44 \mathrm{e}-6$ & $\approx 1.99(2.00)$ \\
& $H^{1}$ & $5.02 \mathrm{e}-2$ & $2.55 \mathrm{e}-2$ & $1.29 \mathrm{e}-2$ & $6.49 \mathrm{e}-3$ & $3.27 \mathrm{e}-3$ & $1.55 \mathrm{e}-3$ & $\approx 1.03(1.00)$ \\
\hline
\end{tabular}

TABLE 10. Numerical results for $u^{r}$ for example (b) with a Riemann-Liouville derivative, by the enriched FEM and $b=1$, $q=x(1-x), \alpha=1.6,1.75,1.9, h=1 / m$.

\begin{tabular}{|c|c|cccccc|c|}
\hline$\alpha$ & $m$ & 10 & 20 & 40 & 80 & 160 & 320 & rate \\
\hline 1.6 & $L^{2}$ & $5.51 \mathrm{e}-4$ & $1.40 \mathrm{e}-4$ & $3.66 \mathrm{e}-5$ & $1.01 \mathrm{e}-5$ & $9.18 \mathrm{e}-6$ & $2.87 \mathrm{e}-6$ & $\approx 1.74(1.70)$ \\
& $H^{1}$ & $2.04 \mathrm{e}-2$ & $1.05 \mathrm{e}-2$ & $5.50 \mathrm{e}-3$ & $2.99 \mathrm{e}-3$ & $1.70 \mathrm{e}-3$ & $1.01 \mathrm{e}-3$ & $\approx 0.78(0.70)$ \\
\hline 1.75 & $L^{2}$ & $3.86 \mathrm{e}-4$ & $9.84 \mathrm{e}-5$ & $2.52 \mathrm{e}-5$ & $6.46 \mathrm{e}-6$ & $1.66 \mathrm{e}-6$ & $4.30 \mathrm{e}-7$ & $\approx 1.96(2.00)$ \\
& $H^{1}$ & $1.38 \mathrm{e}-2$ & $6.98 \mathrm{e}-3$ & $3.55 \mathrm{e}-3$ & $1.79 \mathrm{e}-3$ & $9.04 \mathrm{e}-4$ & $4.51 \mathrm{e}-4$ & $\approx 1.02(1.00)$ \\
\hline 1.9 & $L^{2}$ & $3.00 \mathrm{e}-4$ & $7.52 \mathrm{e}-5$ & $1.88 \mathrm{e}-5$ & $4.71 \mathrm{e}-6$ & $1.18 \mathrm{e}-6$ & $2.94 \mathrm{e}-7$ & $\approx 2.00(2.00)$ \\
& $H^{1}$ & $1.04 \mathrm{e}-2$ & $5.21 \mathrm{e}-3$ & $2.61 \mathrm{e}-3$ & $1.29 \mathrm{e}-3$ & $6.33 \mathrm{e}-4$ & $3.06 \mathrm{e}-4$ & $\approx 1.03(1.00)$ \\
\hline
\end{tabular}

TABLe 11. $\left|\mu-\mu_{h}\right|$ for example (b) with a Riemann-Liouville derivative, by the enriched FEM, and $b=1, q=x(1-x)$, $\alpha=1.6,1.75,1.9, h=1 / m$.

\begin{tabular}{|c|cccccc|c|}
\hline$\alpha$ & 10 & 20 & 40 & 80 & 160 & 320 & rate \\
\hline 1.6 & $4.04 \mathrm{e}-4$ & $1.00 \mathrm{e}-4$ & $2.44 \mathrm{e}-5$ & $5.88 \mathrm{e}-6$ & $1.41 \mathrm{e}-6$ & $3.37 \mathrm{e}-7$ & $\approx 2.07(1.70)$ \\
\hline 1.75 & $1.59 \mathrm{e}-4$ & $3.90 \mathrm{e}-5$ & $9.54 \mathrm{e}-6$ & $2.34 \mathrm{e}-6$ & $5.74 \mathrm{e}-7$ & $1.41 \mathrm{e}-7$ & $\approx 2.02(2.00)$ \\
\hline 1.9 & $8.58 \mathrm{e}-5$ & $2.14 \mathrm{e}-5$ & $5.36 \mathrm{e}-6$ & $1.34 \mathrm{e}-6$ & $3.34 \mathrm{e}-7$ & $8.33 \mathrm{e}-8$ & $\approx 2.00(2.00)$ \\
\hline
\end{tabular}




\section{Conclusions}

In this work, we have developed novel variational formulations for fractional BVPs involving a convection term. The fractional derivative in the leading term is of either Riemann-Liouville or Caputo type. The well-posedness and sharp regularity pickup of the formulations are established. A new finite element method, using continuous piecewise linear finite elements and "shifted" fractional powers for the trial and test space, respectively, was also developed. It leads to a diagonal stiffness matrix for the leading term (on a uniform mesh), and admits optimal $L^{2}(D)$ and $H^{1}(D)$ error estimates, which is the first FEM with such desirable properties. Further, an enriched FEM was proposed to improve the convergence in the Riemann-Liouville case, and optimal error estimates were provided. Extensive numerical experiments fully confirm the convergence analysis.

There are several avenues for further research. First, it is of immense interest to extend the approach to higher dimensions. This extension is formally feasible for the Riemann-Liouville case. However, their solution theory, e.g., well-posedness and sharp regularity pickup, is missing. Second, it is also of much interest to extend the approach to other type or inhomogeneous boundary conditions, which may induce much graver solution singularity. Third, the adaptation of the approach to the time-dependent problems, including the space-time fractional model, is important. Especially, it may allow one to derive optimal $L^{2}(D)$ error estimates.

\section{ACKNOWLEDGEMENTS}

The research of B. Jin has been partly supported by NSF Grant DMS-1319052 and UK EPSRC EP/M025160/1, and R. Lazarov and Z. Zhou was supported in parts by NSF Grant DMS-1016525.

\section{REFERENCES}

[1] R. A. Adams and J. J. F. Fournier. Sobolev Spaces. Elsevier/Academic Press, Amsterdam, second edition, 2003.

[2] B. Baeumer, M. Kovács, and H. Sankaranarayanan. Higher order Grünwald approximations of fractional derivatives and fractional powers of operators. Trans. Amer. Math. Soc., 367(2):813-834, 2015.

[3] D. A. Benson, S. W. Wheatcraft, and M. M. Meerschaert. The fractional-order governing equation of Lévy motion. Water Resour. Res., 36(6):1413-1423, 2000.

[4] Z. Cai and S. Kim. A finite element method using singular functions for the Poisson equation: corner singularities. SIAM J. Numer. Anal., 39(1):286-299, 2001.

[5] S. Chen, J. Shen, and L.-L. Wang. Generalized Jacobi functions and their applications to fractional differential equations. Math. Comp., in press, arXiv:1407.8303, 2014.

[6] D. del-Castillo-Negrete, B. A. Carreras, and V. E. Lynch. Front dynamics in reaction-diffusion systems with Levy flights. Phys. Rev. Lett., 91(1):018302, 4 pp., 2003.

[7] A. Ern and J.-L. Guermond. Theory and Practice of Finite Elements. Springer-Verlag, New York, 2004.

[8] V. J. Ervin and J. P. Roop. Variational formulation for the stationary fractional advection dispersion equation. Numer. Methods Partial Diff. Eq., 22(3):558-576, 2006.

[9] G. J. Fix and J. P. Roop. Least-squares finite element solution of a fractional order two-point boundary value problem. Comput. Math. Appl., 48(7-8):1017-1033, 2004.

[10] R. Gorenflo, Y. Luchko, and M. Yamamoto. Time-fractional diffusion equation in the fractional Sobolev spaces. Fract. Calc. Appl. Anal., 18(3):799-820, 2015.

[11] K. Ito, B. Jin, and T. Takeuchi. On a Legendre tau method for boundary value problems with a Caputo derivative. Fract. Calc. Appl. Anal., page in press, 2015.

[12] B. Jin, R. Lazarov, X. Lu, and Z. Zhou. A simple finite element method for boundary value problems with a Riemann-Liouville derivative. J. Comput. Appl. Math., 293:94-111, 2016. 
[13] B. Jin, R. Lazarov, J. Pasciak, and W. Rundell. Variational formulation of problems involving fractional order differential operators. Math. Comp., 84(296):2665-2700, 2015.

[14] B. Jin, R. Lazarov, J. Pasciak, and Z. Zhou. Error analysis of a finite element method for a space-fractional parabolic equation. SIAM J. Numer. Anal., 52(5):2272-2294, 2014.

[15] B. Jin and Z. Zhou. A finite element method with singularity reconstruction for fractional boundary value problems. ESAIM Math. Model. Numer. Anal., 49(5):1261-1283, 2015.

[16] A. A. Kilbas, H. M. Srivastava, and J. J. Trujillo. Theory and Applications of Fractional Differential Equations. Elsevier, Amsterdam, 2006.

[17] V. E. Lynch, B. A. Carreras, D. del Castillo-Negrete, K. M. Ferreira-Mejias, and H. R. Hicks. Numerical methods for the solution of partial differential equations of fractional order. $J$. Comput. Phys., 192(2):406-421, 2003.

[18] A. C. McBride. Fractional Calculus and Integral Transforms of Generalized Functions. Pitman, Boston, Mass.-London, 1979.

[19] I. Podlubny. Fractional Differential Equations. Academic Press, San Diego, CA, 1999.

[20] A. H. Schatz. An observation concerning Ritz-Galerkin methods with indefinite bilinear forms. Math. Comp., 28:959-962, 1974.

[21] E. Sousa. Finite difference approximations for a fractional advection diffusion problem. $J$. Comput. Phys., 228(11):4038-4054, 2009.

[22] M. Stynes and J. L. Gracia. A finite difference method for a two-point boundary value problem with a Caputo fractional derivative. IMA J. Numer. Anal., 35(2):698-721, 2015.

[23] C. Tadjeran, M. M. Meerschaert, and H.-P. Scheffler. A second-order accurate numerical approximation for the fractional diffusion equation. J. Comput. Phys., 213(1):205-213, 2006.

[24] H. Wang and D. Yang. Wellposedness of variable-coefficient conservative fractional elliptic differential equations. SIAM J. Numer. Anal., 51(2):1088-1107, 2013.

[25] H. Wang, D. Yang, and S. Zhu. Inhomogeneous dirichlet boundary-value problems of spacefractional diffusion equations and their finite element approximations. SIAM J. Numer. Anal., 52(3):1292-1310, 2014.

[26] M. Zayernouri and G. E. Karniadakis. Discontinuous spectral element methods for time- and space-fractional advection equations. SIAM J. Sci. Comput., 36(4):B684-B707, 2014.

Department of Computer Science, University College London, London, WC1E 2BT, UK (BANGTI.JIN@GMAIL.COM,B.JIN@UCL.AC.UK)

Department of Mathematics, Texas A\&M University, College Station, TX 77843-3368 (LAZAROV@MATH.TAMU.EDU)

Department of Applied Physics and Applied Mathematics, Columbia University, 500 W. 120th Street, New York, NY 10027, USA (zhizhou0125@gmail.com) 